\title{
Compensation of Inositol 1,4,5-Trisphosphate Receptor Function by Altering Sarco-Endoplasmic Reticulum Calcium ATPase Activity in the Drosophila Flight Circuit
}

\author{
Santanu Banerjee, Rohit Joshi, Gayatri Venkiteswaran, Neha Agrawal, Sonal Srikanth, Farhan Alam, and Gaiti Hasan \\ National Center for Biological Sciences, Tata Institute of Fundamental Research, Bangalore 560 065, India
}

\begin{abstract}
Ionic $\mathrm{Ca}^{2+}$ functions as a second messenger to control several intracellular processes. It also influences intercellular communication. The release of $\mathrm{Ca}^{2+}$ from intracellular stores through the inositol 1,4,5-trisphosphate receptor $\left(\mathrm{Ins} \mathrm{P}_{3} \mathrm{R}\right)$ occurs in both excitable and nonexcitable cells. In Drosophila, Ins $\mathrm{P}_{3} \mathrm{R}$ activity is required in aminergic interneurons during pupal development for normal flight behavior. By altering intracellular $\mathrm{Ca}^{2+}$ and $\mathrm{Ins}_{3}$ levels through genetic means, we now show that signaling through the $\operatorname{Ins}_{3} \mathrm{R}$ is required at multiple steps for generating the neural circuit required in air puff-stimulated Drosophila flight. Decreased $\mathrm{Ca}^{2+}$ release in aminergic neurons during development of the flight circuit can be compensated by reducing $\mathrm{Ca}^{2+}$ uptake from the cytosol to intracellular stores. However, this mode of increasing intracellular $\mathrm{Ca}^{2+}$ is insufficient for maintenance of flight patterns over time periods necessary for normal flight. Our study suggests that processes such as maintenance of wing posture and formation of the flight circuit require $\operatorname{Ins}_{3}$ receptor function at a slow timescale and can thus be modulated by altering levels of cytosolic $\mathrm{Ca}^{2+}$ and $\mathrm{InsP}_{3}$. In contrast, maintenance of flight patterns probably requires fast modulation of $\mathrm{Ca}^{2+}$ levels, in which the intrinsic properties of the Ins ${ }_{3} \mathrm{R}$ play a pivotal role.
\end{abstract}

Key words: InsP ${ }_{3}$ receptor; SERCA; aminergic interneurons; Gq; phospholipase C; flight patterns

\section{Introduction}

Neuronal control of behavior arises from the activity of underlying neural circuits, which in turn are specified by the interaction of various signaling pathways. Over the past few years, evidence has accumulated demonstrating that ionic calcium $\left(\mathrm{Ca}^{2+}\right)$ plays an important role in the development of activity in neural circuits (Spitzer, 2002; Borodinsky et al., 2004) and hence contributes significantly to the formation of functional neuronal networks. The $\mathrm{Ca}^{2+}$ signal is characterized by a rapid increase in the concentration of free cytosolic calcium $\left(\left[\mathrm{Ca}^{2+}\right]_{\mathrm{i}}\right)$ attributable to the opening of calcium channels located in the plasma membrane and in the membranes of intracellular calcium stores. Free $\mathrm{Ca}^{2+}$ is then rapidly sequestered by the action of calcium pumps and exchangers and also with buffering by cytosolic calcium binding proteins. The functional properties and localization of the channels as well as the pumps, exchangers, and buffers are thought to

Received March 22, 2006; revised July 6, 2006; accepted July 6, 2006.

This work was supported by core grants from the National Center for Biological Sciences and a grant from the Department of Biotechnology to G.H. We thank Aditya Venugopal for PCR mapping of the deletion in $d g q^{221 c}$ as part of an undergraduate training program at the National Centre for Biological Sciences. We are grateful to Prof. V. Rodrigues and K. S. Krishnan for sharing reagents before publication and to Prof. M. K. Mathew for his help with data analysis. We are also grateful to Prof. C.-F. Wu, Dr. J. Lee, Prof. U. Bhalla, and Dr. R. Rajan for their help with the electrophysiology rig and data analysis.

Correspondence should be addressed to Gaiti Hasan, National Center for Biological Sciences, Tata Institute of Fundamental Research, Bellary Road, Bangalore 560 065, India. E-mail: gaiti@ncbs.res.in.

R. Joshi's present address: Department of Biochemistry and Molecular Biophysics, Columbia University Medical Center, HHSC1104, 701 West 168th Street, New York, NY 10032.

S. Srikanth's present address: The Center for Blood Research Institute for Biomedical Research and Department of Pathology, Harvard Medical School, Boston, MA 02115

DOI:10.1523/JNEUROSCI.1231-06.2006

Copyright $\odot 2006$ Society for Neuroscience $\quad$ 0270-6474/06/268278-11\$15.00/0 play a crucial role in generating a range of calcium signals that vary from a brief local increase to repetitive calcium spikes and waves spreading over a larger region. The kinetics, amplitude, and duration of these signals in turn determine the cellular specification of neurotransmitters, receptors, and channels that influence synaptic activity and plasticity in cognate neurons (Berridge, 1998; Spitzer et al., 2000).

Our goal is to understand the contribution of the intracellular $\mathrm{Ca}^{2+}$ release channel, the Ins $\mathrm{P}_{3}$ receptor $\left(\mathrm{InsP}_{3} \mathrm{R}\right)$, in the development and function of neural circuitry. From studies in Drosophila, we have shown that flight is critically dependent on normal activity of the $\operatorname{Ins}_{3} \mathrm{R}$ in aminergic interneurons during pupal development suggesting that InsP ${ }_{3}$-mediated $\mathrm{Ca}^{2+}$ release is required during normal development of the flight circuit (Banerjee et al., 2004). Mutants in the gene encoding the $\mathrm{InsP}_{3} \mathrm{R}$ (itpr) in Drosophila exhibit a range of defects including altered wing posture, increased spontaneous firing, and loss of rhythmic flight patterns in response to an air puff stimulus. Together, these phenotypes contribute to the loss of flight behavior observed in itpr mutants. An obvious question that arises from these studies is whether the multiple phenotypes arise as a consequence of a single early neuronal dysfunction. Alternately, each phenotype could be attributable to independent events requiring $\operatorname{Ins}_{3} \mathrm{R}$ activity at individual and distinct steps of flight circuit development. Here, we have addressed these questions by altering intracellular $\mathrm{Ca}^{2+}$ signals in flight-deficient itpr mutants through genetic means. In the first instance, a dominant mutant for the sarco-endoplasmic reticular $\mathrm{Ca}^{2+}$-ATPase (SERCA) pump was introduced into itpr mutant backgrounds. SERCA is responsible 
for pumping cytosolic $\mathrm{Ca}^{2+}$ into the endoplasmic reticulum (ER) store and thus maintaining the intracellular concentrations of $\mathrm{Ca}^{2+}$ both in the cytosol and in the ER store. The second class of mutants are in genes that encode (1) the $\alpha$ subunit of the heterotrimeric G-protein G $\alpha$ q (Scott et al., 1995; Scott and Zuker, 1998) and (2) phospholipase C $\beta$ (PLC $\beta$ ) (Bloomquist et al., 1988; Shortridge et al., 1991). These mutants are expected to reduce Ins $\mathrm{P}_{3}$ levels and thus reduce the activity of the Ins $\mathrm{P}_{3} \mathrm{R}$, in circumstances in which $\mathrm{InsP}_{3}$ is generated by the activation of seven transmembrane receptors. Our results show that neuronal phenotypes of itpr mutants arise from at least two distinct classes of intracellular $\mathrm{Ca}^{2+}$ signals. One class can be modulated by the strength of $\mathrm{InsP}_{3}$ signaling and the rate of $\mathrm{Ca}^{2+}$ uptake into the ER. The second class appears to depend primarily on the $\mathrm{Ca}^{2+}$ release properties of the $\mathrm{InsP}_{3}$ receptor.

\section{Materials and Methods}

Drosophila strains. Different mutant alleles for the itpr gene were tested for flight. The viable heteroallelic combinations used in this study were $i t p r^{w c 703 / w c 361}, i t p r^{\text {ka1091/ug3 }}$. These are single point mutants in the itpr gene that were generated in an EMS (ethyl methanesulfonate) screen. Detailed molecular information on these alleles has been published recently (Joshi et al., 2004; Srikanth et al., 2004b). For rescue experiments, embryonic wild-type itpr cDNA (UASitpr ${ }^{+}$) (Venkatesh et al., 2001) and $\alpha 3$ splice variant of the $d g q$ gene (UASdgq $\alpha 3^{+}$) (Ratnaparkhi et al., 2002) were used. $\mathrm{Ca}-\mathrm{P} 60 \mathrm{~A}^{\text {Kum170ts }}$ (referred as Kum ${ }^{170}$ throughout the text) was obtained from Dr. K. S. Krishnan (Sanyal et al., 2005a), $d g q^{221 c}$ was generated by Pinky Kain in collaboration with Veronica Rodrigues, whereas $d g q^{18745}$ was procured from the Bloomington Stock Center (Bloomington, IN). plc21 $c^{P 319 / 11}$ (referred to as $p l c 21 c^{P 319}$ in this study) was obtained from Dr. S. Leevers (Weinkove et al., 1999), and norpA $A^{P 24}$ is a null allele of norpA gene (Pearn et al., 1996). DdcGAL4 (Li et al., 2000), and $h s p 70 G A L 4$ strains [referred to as $h s G A L 4^{L}$ in Banerjee et al. (2004)] were obtained from the Bloomington Stock Center. $h s G A L 4^{L}$ exhibits basal green fluorescent protein (GFP) expression at $25^{\circ} \mathrm{C}$, when crossed to UASGFP. Because the UASGq3 strain described previously (Ratnaparkhi et al., 2002) was lost, a similar strain was remade. In the newly generated transgenic $U A S G q 3$ flies (referred to as $U A S d g q \alpha 3^{+}$), the insert was mapped to chromosome 2 . The following fly strains were generated by standard genetic methods using individual mutant and transgenic fly lines described above: (1) Kum ${ }^{170} / \mathrm{CyoG}$;itpr ${ }^{\text {ug3 }} / \mathrm{TM} 6 \mathrm{~Tb}$, (2) $U_{\text {USitpr }}^{+} ;+/+; i t p r^{k a 1091} /$ TM6Tb, (3) UASitpr ${ }^{+} ;+/+; i t p r^{w c 703} / T M 6 T b$, (4) ElavGAL4/CyoG;itpr $r^{u g 3} / T M 6 T b$, (5) $d g q^{221 c} / C y o G$; itpr $r^{w c 703} / T M 6 T b$, (6) $d g q^{18745} /$ CyoG;itpr ${ }^{w c 703} / T M 6 T b$, (7) plc21c ${ }^{P 319} ; i t p r^{w c 703} / T M 6 T b,(8)$

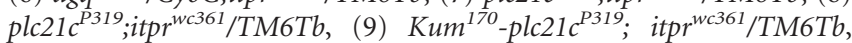
(10) $h s G A L 4^{L}-d g q^{221 c} / C y o G$; itpr $r^{w c 361} / T M 6 T b,(11)$ DdcGAL4- $d g q^{221 c}$ / CyoG; itpr $r^{w c 361} / T M 6 T b$, (12) UASdgq $\alpha 3^{+} / C y o G$; itpr $r^{w c 703} / T M 6 T b$, and (13) $U A S d g q^{1 f 1} ;$ itpr $^{w c 703} /$ TM6Tb.

In the above description of genetic strains and in the rest of the text, the symbol "/" separates two homologous chromosomes, ";" separates two different chromosome, and “-” designates alleles of two genes or transgenes recombined onto one chromosome.

Larval staging and lethality measurements. To obtain molting profiles of heteroallelic mutant larvae, staging experiments were performed as described previously (Joshi et al., 2004). Heteroallelic and heterozygous larvae were identified based on the Tubby phenotype, which is visible in larvae from $60 \mathrm{~h}$ after egg laying (AEL). Timed and synchronized egg collections were done for a period of $6 \mathrm{~h}$ at $25^{\circ} \mathrm{C}$. The cultures were then allowed to grow at 17.5 or $25^{\circ} \mathrm{C}$ depending on the experiment. The temperature of $17.5^{\circ} \mathrm{C}$ was chosen because larval development takes precisely double the time at this temperature compared with development at $25^{\circ} \mathrm{C}$. Heteroallelic mutant larvae were selected at 56-64 h AEL when the experiments were done at $25^{\circ} \mathrm{C}$ and at an interval of $116-124 \mathrm{~h}$ AEL in cases in which the experiments were done at $17.5^{\circ} \mathrm{C}$. Larvae were selected based on the dominant markers Tubby (on TM6Tb) and CyoGFP and transferred into vials of cornmeal medium lacking agar. These larvae were grown at the desired temperatures of 17.5 or $25^{\circ} \mathrm{C}$ and screened at ap- propriate time points, for number of survivors and their stage of development. Larval stages were determined by the morphology of the anterior spiracles (Ashburner, 1989). For each time interval, a minimum of 150 larvae were screened in batches of 50 larvae each.

Flight assay. Flight tests were performed as described by Banerjee et al. (2004) following minor modifications of the "cylinder drop assay" described previously (Benzer, 1973). A batch of 20 flies was tested each time. A minimum of five batches were tested for each genotype. Flies that dropped directly down the cylinder were collected in a vial kept on ice underneath the lower opening of the cylinder. These were counted as flight defective. Flies that were able to hold onto the walls of the cylinder were considered as fliers. The percentage of flight-defective organisms was determined as $F / T \times 100$, where $F$ is the number of flies that dropped into the vial at the bottom of the cylinder and $T$ is the total number of flies tested. Computation of means, SDs, and $t$ tests was performed using Origin software (Origin Lab, Northampton, MA).

Electrophysiological preparation and recordings. Physiological recordings were performed on the dorsal longitudinal muscles (DLMs) of the giant fiber pathway (Tanouye and Wyman, 1980). Recording methods for the muscle have been described previously (Engel and Wu, 1992; Lee and $\mathrm{Wu}, 2002)$. For measurement of responses to an air puff stimulus, flies were anesthetized briefly with diethyl ether and glued to a thin metal wire between the neck and the thorax with nail polish. Flies were allowed to recover from anesthesia for $\sim 4 \mathrm{~h}$. After recovery, an uninsulated tungsten electrode $(0.5 \mu \mathrm{m})$, which had been sharpened by electrolysis, was inserted carefully into the DLM (fiber a) just beneath the cuticle. A similar tungsten electrode was inserted in the abdomen as a reference. Flies were rested for at least $10 \mathrm{~min}$ after insertion of electrodes before beginning the recording. For measurement of spontaneous activity, flies were kept undisturbed and recordings were performed for $2 \mathrm{~min}$. To record air puff responses, a gentle mouth-blown air puff stimulus was delivered to the fly kept in a tethered condition. Responses were measured from DLM "a" with a tungsten electrode (specification mentioned above), using an ISODAM8A (World Precision Instruments, Sarasota, FL) amplifier with filter set up $30 \mathrm{~Hz}$ (low pass) to $>10 \mathrm{kHz}$ (high pass). Gap free mode of pClamp8 (Molecular Devices, Union City, CA) was used to digitize the data $(10 \mathrm{kHz})$ on a Pentium 5 computer equipped with Digidata 1322A (Molecular Devices). Data were analyzed using Clampfit (Molecular Devices) and plotted using Origin (MicroCal, Northampton, MA).

Microsomal vesicle $\mathrm{Ca}^{2+}$ release assay. A modified protocol from Bramley et al. (1990) was used, which has been described recently (Srikanth et al., 2004a). In brief, microsomes were prepared in the presence of $200 \mu \mathrm{M}$ free $\mathrm{Ca}^{2+}$, from either 75 wandering third-instar larvae or $\sim 200$ adult heads, obtained from the appropriate genotypes. Microsomes were made in parallel, in $\mathrm{Ca}^{2+}$-free buffer and otherwise identical conditions, and added to the buffers used for determining the standard curve for $\mathrm{Ca}^{2+}$ for each individual experiment. Membrane-impermeant $\mathrm{Ca}^{2+}$-sensitive ratiometric fluorescent dye, Fura-2 (Invitrogen, Eugene, OR) was prepared in calcium-free water. Steady-state fluorescence measurements were performed in a SPEX Fluorolog-2 spectrofluorometer (SPEX Industries, Edison, $\mathrm{NJ}$ ) at $20^{\circ} \mathrm{C}$ so as to minimize nonspecific calcium leak. For each run, $\sim 15 \mu \mathrm{g}$ of adult head microsomes were added to $2 \mathrm{ml}$ of assay buffer (20 mm Tris, $\mathrm{pH} 7.4$, and $80 \mathrm{~mm} \mathrm{NaCl}$ in calcium-free water) containing $5 \mu \mathrm{M}$ Fura-2. Steady-state kinetics of $\mathrm{Ca}^{2+}$ release were measured at various concentrations of $\operatorname{Ins}_{3}$ and quantified by plotting a standard curve with known amounts of free $\mathrm{Ca}^{2+}$ using the standard $\mathrm{Ca}^{2+}$-EGTA buffering system.

Neuron culture and calcium imaging. Primary cultures of Drosophila larval neurons were plated in $200 \mu \mathrm{l}$ of Schneider's medium supplemented with $10 \%$ fetal bovine serum (Invitrogen), $50 \mathrm{U} / \mathrm{ml}$ penicillin, 50 $\mu \mathrm{g} / \mathrm{ml}$ streptomycin, and $10 \mu \mathrm{g} / \mathrm{ml}$ Amphotericin B as described previously (Wu et al., 1983). Briefly, brain and the ventral ganglion complex were dissected from surface-sterilized Drosophila third-instar larvae of the appropriate genotypes. The brain tissue was mechanically dissociated using syringe needles in Schneider's medium containing collagenase $(0.75 \mu \mathrm{g} / \mu \mathrm{l})$ and dispase $(0.4 \mu \mathrm{g} / \mu \mathrm{l})$ and incubated in the proteolytic medium for $1 \mathrm{~h}$ to allow complete dissociation of the tissue. The lysate containing essentially single cells was then spun down, resuspended in Schneider's medium (200 $\mu \mathrm{l}$ of the medium was added for every four 
brains dissected), and plated onto $35 \mathrm{~mm}$ culture dishes with a poly-Llysine-coated coverslip for the bottom. The cells were incubated at $22^{\circ} \mathrm{C}$ for $14-16 \mathrm{~h}$ before imaging.

Calcium imaging in larval neurons. Larval neuron cultures were washed twice after growth for 14-16 h with Drosophila M1 medium $(20 \mathrm{~mm}$ HEPES, $150 \mathrm{~mm} \mathrm{NaCl}, 5 \mathrm{~mm} \mathrm{KCl}, 1 \mathrm{~mm} \mathrm{MgCl}, 1 \mathrm{~mm} \mathrm{CaCl}_{2}$, and sucrose, $\mathrm{pH}$ 6.9). They were loaded in the dark with $2.5 \mu \mathrm{M}$ Fluo-3AM (see Fig. $2 A a, A b)$ or Fluo-4AM $(A c)$ in M1 medium containing $0.002 \%$ Pluronic F-127 for $30 \mathrm{~min}$ at room temperature. The fluorescent dyes were obtained from Invitrogen. After washing three times with M1, the cells were finally covered with $100 \mu \mathrm{l}$ of M1 and imaged within 40 min of loading. For quantitative analysis, a field with several cells was selected and imaged using the epifluorescence optics of a Nikon TE2000 inverted widefield microscope with an oil objective ( $60 \times$ and 1.4 numerical aperture) lens. Excitation of Fluo-3/4 was performed using $488 \mathrm{~nm}$ wavelength illumination from a mercury arc lamp. Emitted light was detected through a $505 \mathrm{~nm}$ bandpass filter (FITC filter set, 41001-exciter HQ480/ 40, dichroic Q505LP, emitter HQ535/50; Chroma, Brattleboro, VT). Image acquisition was performed using the Evolution QEi CCD camera and QED imaging software (Media Cybernetics, Silver Spring, MD). The time lapse acquisition mode of the software was used to follow fluorescence changes in the cells every $5 \mathrm{~s}$ for a period of $1 \mathrm{~min}$. Each exposure was for $200 \mathrm{~ms}$. The cells were depolarized with $70 \mathrm{~mm} \mathrm{KCl}$ within the first $5 \mathrm{~s}$ after the start of data acquisition. As a control, a series of images were acquired under the same imaging protocol without addition of $\mathrm{KCl}$. A total of $\sim 70$ cells was analyzed from 10-15 dishes imaged for each genotype. Finally, $\sim 45$ cells for each genotype were selected for the graph in Figure $2 A c$. The cells were selected based on the observation that the $\mathrm{Ca}^{2+}$ signal decayed following the expected first-order exponential decay kinetics.

Data analysis. For measuring increase in fluorescence with time, images were processed using the NIH ImageJ software, version 1.33. Fluorescence intensity before $\left(F_{\text {basal }}\right)$ and after depolarization $\left(F_{\max }\right)$ with $\mathrm{KCl}$, was determined for all of the time points shown in Figure $2 \mathrm{~A}$. Background fluorescence (an area without any cells) was subtracted from the fluorescence values for each cell. The data were plotted using Origin 6.0 software as follows: $\Delta F / F=F_{\max }-F_{\text {basal }} / F_{\text {basal }}$ for each cell. The mean and SE for $\Delta F / F$ values of the cells were calculated for every time point and compared between two appropriate genotypes using the Student's $t$ test. The mean values for each time point were normalized to the mean peak value reached for each individual genotype taken as 100 . No significant difference was observed between the mean peak values of the six genotypes tested.

Western blots. Protein extracts from $\sim 100$ first-instar larvae (aged $28-36 \mathrm{~h}$ after egg laying) of indicated genotype were made by homogenization in $100 \mu \mathrm{l}$ of homogenizing buffer containing 50 mм Tris, $\mathrm{pH} 8.0$, $150 \mathrm{~mm} \mathrm{NaCl}, 1$ mм EGTA, 1.5 mm MgCl 2 , Triton X-100 (1\%), 6 м urea. Third-instar larvae (10-15) were used for making lysates from $d g q^{221 c /+}$, $d g q^{18745 /+}$, Arm GAL4/UASdgq $q^{1 f 1}$, and wild-type animals. The samples were run on a $12 \%$ SDS-polyacrylamide gel and transferred to nitrocellulose membranes by standard Western blotting protocols. Anti-Gq antiserum from Santa Cruz Biotechnology (SC-3912; Santa Cruz, CA) was used at a dilution of 1:500. Secondary anti-rabbit (donkey) antibody was used at 1:10,000 dilution. To estimate amount of protein loaded, $\beta$-tubulin levels were detected using anti- $\beta$-tubulin (E7; Developmental Studies Hybridoma Bank, Iowa City, IA) at a dilution of 1:330 and an anti-mouse secondary (catalog \#7076; Cell Signaling Technology, Beverly, MA) was used at a dilution of 1:1000. Secondary antibodies were conjugated to horseradish peroxidase, and the detection of protein in the blot was done by addition of a chemiluminescence substrate from Pierce (catalog \#34075; Rockford, IL). Typically, 1-2 s exposures were given to develop the blot.

PCR mapping of dgq coding region deletion in $\mathrm{dgq} 221 \mathrm{c}$. Genomic DNA was isolated from first-instar larvae of the appropriate genotypes mentioned in Figure $3 B$ according to standard protocol. Amplification of three fragments (see Fig. 3B, denoted as F1, F2, F3) was by following standard PCR protocols. Primers used for amplification of the F1 fragment are P11 (5'-AACCATCACTCTCAGC-3') and P12 (5'-GCACTCCATGCTAACTA-3'), for F2 are P1 (5' -AGCGGTTACTCGGACGA-
GGACA-3') and P3 (5'-CTCAAGAATGCCAGTTGTCGGCAC-3'), and for $\mathrm{F} 3$ are P17 (5'-GTGGTCAGCGATCCGAG-3') and P8 ( $5^{\prime}$-GCACACGTGAAATGAGAATAGA- $\left.3^{\prime}\right)$.

Generation of a dsRNA construct for the dgq gene. The $d g q \alpha 3 \mathrm{cDNA}$ isolated from an appendage cDNA library (Ratnaparkhi et al., 2002) was used as a template to generate an inverted repeat construct coding for a dsRNA, which would encompass a region common to all $d g q$ splice variants (approximate position of the primers used is shown in Fig. $3 A$ ). Briefly, two PCR products were created of $\sim 770$ from dgq $\alpha 3$ cDNA. The first product was created using a $5^{\prime}$ primer containing $K p n I$ site $\left(5^{\prime}\right.$ GGTACC(538)TCACGATACTAGCAGCATCCC-3') and a $3^{\prime}$ primer containing a BamH1 site (5'-GGATCC(1314)CGGTGTAAGCGAGCGAAG- $\left.3^{\prime}\right)$ at the ends of the respective primers. This product was then cloned into the KpnI and BamH1 of pUAST (Brand et al., 1994). A linker region of 12 nucleotides was introduced during the PCR [ 6 bp overhang from the cDNA (1308-1314) and 6 bp from the BamH1 site]. A second PCR product was created using a reverse $5^{\prime}$ primer containing an $\mathrm{XbaI}$ site (5'-TCTAGA(539)GTGCTATGATCGTCGTAGGGA-3') and a reverse $3^{\prime}$ primer containing a BamH1 site (5'-GGATCC(1308)TTCGCTCGCTTCTCAATTCT- $3^{\prime}$ ) at the $5^{\prime}$ end. This was cloned in the pUAST KpnI-Bam H1 constituent (containing the previous insert) using $B a m \mathrm{H} 1$ and $\mathrm{XbaI}$ sites, thus creating an inverted repeat. Germline transformants with this construct were obtained following the standard protocol of Drosophila embryonic microinjection.

\section{Results}

\section{Differential suppression of phenotypes arising from mutant InsP $\mathrm{P}_{3}$ Rs by a dominant mutant in dSERCA or CaP60A}

Among the existing set of itpr mutant alleles, are a series of heteroallelic itpr mutant animals, which when grown at $25^{\circ} \mathrm{C}$ exhibit altered wing posture and severe flight defects. Interestingly, these heteroallelic itpr mutants are lethal when grown at $17.5^{\circ} \mathrm{C}$ (Joshi et al., 2004). These mutant combinations thus serve as a sensitized genetic background that can be used for assessing the role of modifiers of $\mathrm{InsP}_{3} \mathrm{R}$ phenotypes. The identification of such modifiers is likely to lead to a better understanding of how $\mathrm{InsP}_{3}$ mediated $\mathrm{Ca}^{2+}$ release modulates neuronal function. Among these heteroallelic mutant combinations, one of the best studied is itprka1091/ug3 (Banerjee et al., 2004; Joshi et al., 2004). A change in the $\mathrm{Ca}^{2+}$ release properties of $i t p r^{k a 1091 / u g 3}$ channels was first identified by measuring the kinetics of $\mathrm{Ca}^{2+}$ release in response to $\mathrm{InsP}_{3}$ in microsomal vesicles obtained from itpr $\mathrm{ka1091/ug3}^{\mathrm{or}-}$ ganisms and compared with microsomal vesicles from wild-type heads (Fig. 1A). Decreased levels of $\mathrm{Ca}^{2+}$ release was observed after InsP $\mathrm{P}_{3}$ stimulation of microsomes prepared from either adult heads or larvae of $i t p r^{k a 1091 / u g 3}$ when compared with microsomal vesicles from wild-type animals of a comparable developmental stage. These in vitro data suggest that it might be possible to suppress phenotypes associated with itpr $r^{k a 1091 / u g 3}$ in vivo by elevating $\left[\mathrm{Ca}^{2+}\right]_{\mathrm{i}}$ through genetic means. One way to achieve this would be to reduce the uptake of cytosolic $\mathrm{Ca}^{2+}$ into intracellular stores. Toward this end, a dominant mutant allele $\left(\mathrm{Kum}^{170}\right)$ for the gene $(\mathrm{Ca}-\mathrm{P} 60 \mathrm{~A})$ encoding the SERCA, was introduced in itpr $r^{\text {ka1091/ug3 }}$ organisms.

Although $\mathrm{Kum}^{170}$ was originally isolated as a temperaturesensitive dominant paralytic whose focus lay in muscle tissue (Sanyal et al., 2005a), recent work has established it as a dominant mutant that affects SERCA function at permissive temperatures also (Sanyal et al., 2005b). To examine the effect of $\mathrm{Kum}^{170}$ on $\mathrm{itpr}$ mutant phenotypes, animals of the genotype $\mathrm{Kum}^{170 /+}$; itpr $^{\mathrm{ka1091/ug3}}$ were grown at either 25 or $17.5^{\circ} \mathrm{C}$. Interestingly, the presence of a single copy of $\mathrm{Kum}^{170}$ could suppress the altered wing posture seen in itpr ${ }^{\text {ka1091/ug } 3}$ organisms at $25^{\circ} \mathrm{C}$ (Fig. 1 B). The suppression was not evident in $\mathrm{Kum}^{170 /+} ;$ itpr $^{\text {ka1091/ug3 }}$ flies grown at $17.5^{\circ} \mathrm{C}$. However, $\mathrm{Kum}^{170}$ could effectively suppress larval lethality seen 


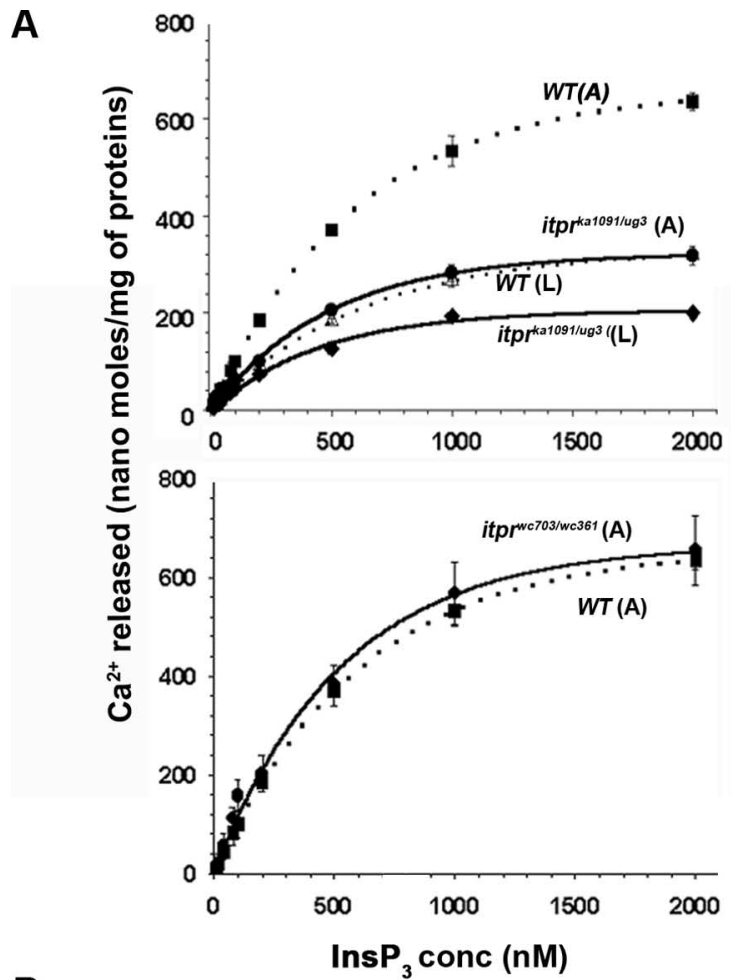

B

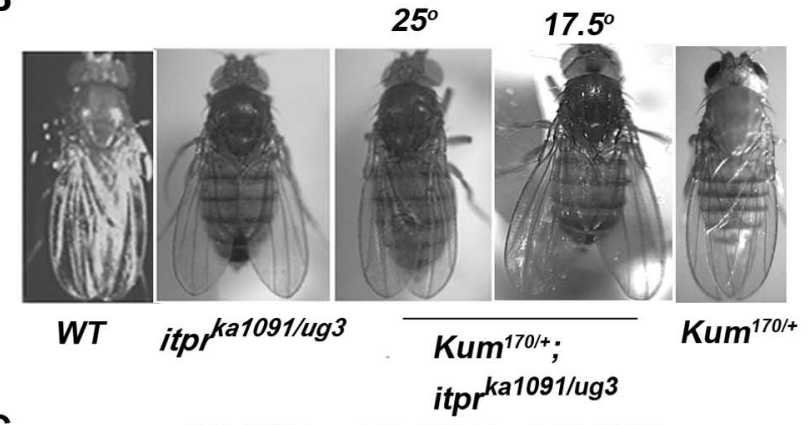

C

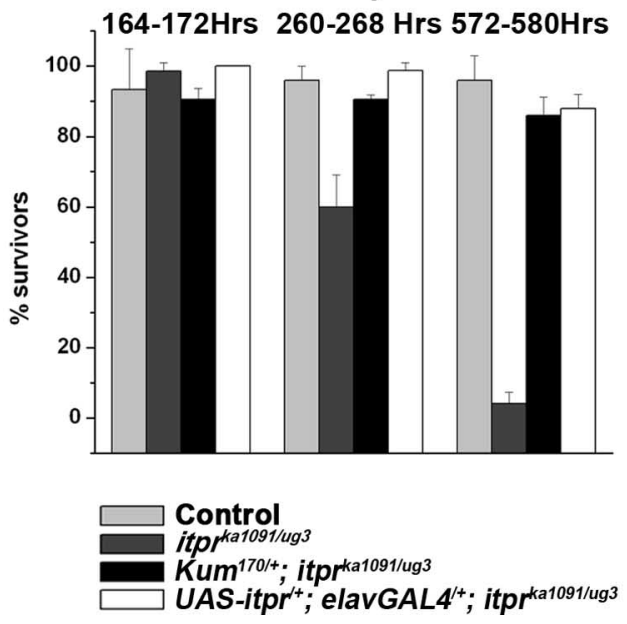

Figure 1. itpr mutant phenotypes can be suppressed by a dominant mutation, $\mathrm{Kum}^{170}$, in the Ca-P60A gene. $A$, Microsomal vesicles derived from itpr ${ }^{\text {ka1091/ug3 }}$ animals (solid line) release lower levels of $\mathrm{Ca}^{2+}$ in response to exogenously added InsP ${ }_{3}$, compared with microsomal vesicles derived from wild-type animals (dashed line) as shown in the top panel ( $A$, microsomes prepared from adult head; $\mathrm{L}$, microsomes prepared from larvae). In contrast, Ins $\mathrm{P}_{3}$-stimulated $\mathrm{Ca}^{2+}$ release from vesicles prepared from itpr ${ }^{w c 703 / w c 361}$ animals appeared similar to those prepared from wild-type animals. Western blot experiments performed with vesicles derived from each genotype exhibit equivalent levels of the $\operatorname{lns}_{3} R$ protein in all three genotypes (data not shown). $\boldsymbol{B}$, Kum $^{170 /+} ;$ itpr $^{\mathrm{ka1091/ug3}}$ animals grown throughout at $25^{\circ}$ C exhibit normal wing posture compared with itpr ${ }^{k a 1091 / u g 3}$ animals in which the wings are spread out. This phenotype in itpr $r^{k a 1091 / u g 3}$ animals at $17.5^{\circ} \mathrm{C}$ (Fig. $1 C$ ), indicating that the $\mathrm{Kum}^{170}$ allele exerts its mutant effect at temperatures as low as $17.5^{\circ} \mathrm{C}$. The cellular focus of $i t p r$ mutant lethality at $17.5^{\circ} \mathrm{C}$ lies in neurons, because expression of an $\mathrm{itpr}^{+}$transgene under the control of a pan-neuronal promoter rescues it completely (Fig. 1C). We therefore tested whether there was an effect of the Kum ${ }^{170}$ allele on bringing cytosolic $\mathrm{Ca}^{2+}\left(\left[\mathrm{Ca}^{2+}\right]_{\mathrm{i}}\right)$ back to basal levels in larval neurons after a depolarization stimulus. As shown in Figure $2 \mathrm{~A}$, larval neurons cultured from $\mathrm{Kum}^{170 /+}$ animals return to basal levels of $\left[\mathrm{Ca}^{2+}\right]_{\mathrm{i}}$ at a rate slower than what is observed for neurons from wild-type larvae, after depolarization by the addition of $70 \mathrm{~mm} \mathrm{KCl}$. Thus, in agreement with previous data from cardiac and muscle cells, in neurons too the Kum ${ }^{170}$ mutation in SERCA slows down the rate of $\mathrm{Ca}^{2+}$ entry into intracellular stores. We have not confirmed whether the change in this rate depends on temperature, but based on the differential suppression of neuronal phenotypes at 17.5 and $25^{\circ} \mathrm{C}$ described above, this seems likely.

We then tested the effect of $\mathrm{Kum}^{170}$ on calcium sequestration in larval neurons from itpr mutants after $\mathrm{KCl}$ depolarization. The rate of return to basal $\left[\mathrm{Ca}^{2+}\right]_{\mathrm{i}}$ is similar to wild type in both $i t p r$ mutants tested (itpr ${ }^{k a 1091 / u g 3}$ and $i t p r^{w c 361 / w c 703}$ ), whereas it is slower (and comparable with $\mathrm{Kum}^{170 /+}$ ) in $\mathrm{Kum}^{170 /+}$; itpr $^{\text {kalogl/ug } 3}$ and $\mathrm{Kum}^{170 /+}$; $i \mathrm{tpr} \mathrm{r}^{w c 361 / w c 703}$ neurons (Fig. $2 \mathrm{Ac}$ ). The $\mathrm{InsP}_{3} \mathrm{R}$ is known to alter $\left[\mathrm{Ca}^{2+}\right]_{\mathrm{i}}$ only in response to the generation of intracellular $\mathrm{InsP}_{3}$. Any change in cytosolic sequestration in $\mathrm{Ins}_{3} \mathrm{R}$ mutants on depolarization with $\mathrm{KCl}$ would very likely reflect a change in the basal concentration of store calcium. Our data do not support this idea.

Suppression of larval lethality (Fig. 1C) and wing posture by $\mathrm{Kum}^{170}$ led us to examine the status of other neuronal phenotypes associated with itpr mutants (Banerjee et al., 2004; Joshi et al., 2004). Initially, the flight behavior of $\mathrm{Kum}^{170 /+} ; \mathrm{itpr}^{\mathrm{ka1091/ug3} 3}$ animals was compared with that of $i \mathrm{tpr}^{\mathrm{ka1091/ug} 3}$. The percentage of organisms with flight defects was no different between the two strains, when measured in a "cylinder drop test" assay (Fig. 2B). Most simplistically, these data indicate that itpr mutant phenotypes of larval lethality and wing posture on one hand and flight behavior on the other hand, arise from differential intracellular calcium requirements. This idea is strongly supported by results of the next set of experiments, which looked at the status of spontaneous firing and air puff-induced flight patterns from the indirect flight muscles in $\mathrm{Kum}^{170 /+}$; itpr $r^{\text {kalog1/ug } 3}$ organisms.

In wild-type flies, recording of spontaneous activity from the dorsal longitudinal flight muscles reveals occasional bursts of action potentials. itpr mutants display a considerably higher frequency of such spontaneous action potentials (Banerjee et al., 2004). In $\mathrm{Kum}^{170 /+}$; $i t \mathrm{pr}^{\mathrm{ka1091/ug3}}$ organisms (grown at $25^{\circ} \mathrm{C}$ ), spontaneous firing from the DLMs was significantly reduced compared with itpr ${ }^{k a 1091 / u g 3}$ animals (Fig. 2C,D). No obvious effect on spontaneous firing is observed in $\mathrm{Kum}^{170 /+}$ animals at $25^{\circ} \mathrm{C}$ (Fig. $2 C, D$ ). Thus, the Kum ${ }^{170 /+}$ mutant allele can suppress this aspect of flight physiology despite its inability to suppress flight behavior in the cylinder drop test. Next, we tested the flight response to a gentle air puff in these flies. Unlike itpr $r^{k a 1091 / u g 3}$ flies, which are unable to initiate any rhythmic flight patterns, the

$\leftarrow$

is not suppressed in $\mathrm{Kum}^{170 /+} ;$ itpr ${ }^{\mathrm{ka1091/ug3}}$ animals when grown at $17.5^{\circ} \mathrm{C}$. The wing posture of Kum ${ }^{170 /+}$ animals at 25 and $17.5^{\circ} \mathrm{C}$ appears like wild type. C, Animals of Kum ${ }^{170 /+} ;$; itpr $r^{\text {Ka0997/ug } 3}$ grown at $17.5^{\circ} \mathrm{C}$ after egg laying at $25^{\circ} \mathrm{C}$ have an improved survival profile compared with itpr $^{\text {Ka1091/ug3 }}$ animals grown under the same conditions. Kum ${ }^{170 /+}$ larvae are fully viable at both 25 and $17.5^{\circ} \mathrm{C}$ (data not shown). Error bars indicate SEM. 
$\mathrm{Kum}^{170 /+}$; itpr ${ }^{k a 1091 / u g 3}$ animals exhibit a few cycles of wing beating when stimulated with an air puff. Simultaneous recordings made from the DLMs, showed the rhythmic generation of action potentials immediately after the air puff delivery (Fig. 2E; supplemental movie 1, available at www.jneurosci.org as supplemental material). However, unlike wild-type flight patterns, the air puff response in $\mathrm{Kum}^{170 /+}$; itpr $^{\mathrm{ka1091/ug} 3}$ terminated in $\sim 5$ s. The Kum ${ }^{170}$ mutant allele of SERCA can thus partially suppress the inability of $i t p r$ mutants to respond to an air puff.

The differential suppression of these neuronal phenotypes by $\mathrm{Kum}^{170}$ could arise from a difference in the level of $\left[\mathrm{Ca}^{2+}\right]_{\mathrm{i}}$ required for suppression of each of these phenotypes. We therefore tested suppression of flight behavior in another itpr heteroallelic combination, $\operatorname{itpr}^{w c 703 / w c 361}$, in which flight defects are limited to $30 \%$ as measured by the cylinder drop test assay (Banerjee et al., 2004) and in which release of intracellular $\mathrm{Ca}^{2+}$ on $\mathrm{InsP}_{3}$ stimulation is not observably reduced as judged by the microsomal vesicle assay (Fig. 1A). Here, too, the introduction of a single copy of $\mathrm{Kum}^{170}$ did not suppress defects in flight behavior (Fig. 2B). The nonfliers from among itpr ${ }^{w c 703 / w c 361}$ animals exhibit brief rhythmic flight patterns that are usually not sustained beyond $5 \mathrm{~s}$. In organisms of the genotype $\mathrm{Kum}^{170 /+}$; itpr $r^{\text {wc361/wc703, }}$ flight physiology was no different from $i_{\text {tp }}{ }^{\text {wc361/wc703 }}$ flies (data not shown). Thus, whereas $\mathrm{Kum}^{170}$ can suppress $\mathrm{InsP}_{3} \mathrm{R}$ mutant phenotypes of larval viability, wing posture, spontaneous firing, and initiation of the air puff response, it is unable to suppress maintenance of flight patterns for periods beyond $5 \mathrm{~s}$. The latter phenotype remains unchanged in itpr mutants with subnormal $\left(\right.$ itpr $^{\mathrm{ka1091/ug3}^{3}}$ ) or near-normal $\left(\right.$ itpr $\left.^{w c 361 / w c 703}\right)$ levels of $\mathrm{Ca}^{2+}$ release, suggesting that it is the nature of the $\mathrm{Ca}^{2+}$ signal rather than levels of intracellular $\mathrm{Ca}^{2+}$ that dictates the maintenance of flight patterns.

The precise change in the nature of the $\mathrm{Ca}^{2+}$ signal in $i_{p} r^{w c 703 / w c 361}$ organisms remains to be understood. However, based on our previous findings with WC703 single channels, it is expected that the $\mathrm{Ca}^{2+}$ dependence of $\mathrm{InsP}_{3}$-mediated $\mathrm{Ca}^{2+}$ release from WC703/WC361 channels will be altered in vivo. Whereas wild-type Drosophila $\mathrm{InsP}_{3}$ receptors are activated and inhibited over a wide range of $\left[\mathrm{Ca}^{2+}\right]$, WC703 channels exhibit this activation and inhibition over a very narrow range of calcium concentrations (Srikanth et al., 2004a). These data support the idea that $\mathrm{Ca}^{2+}$ release through the Ins $\mathrm{P}_{3} \mathrm{R}$ results in at least two kinds of intracellular $\mathrm{Ca}^{2+}$ signals. This idea was tested further by analyzing the effect of mutants predicted to reduce $\mathrm{Ins}_{3}$ signaling.

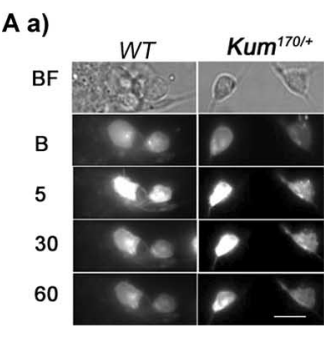

B

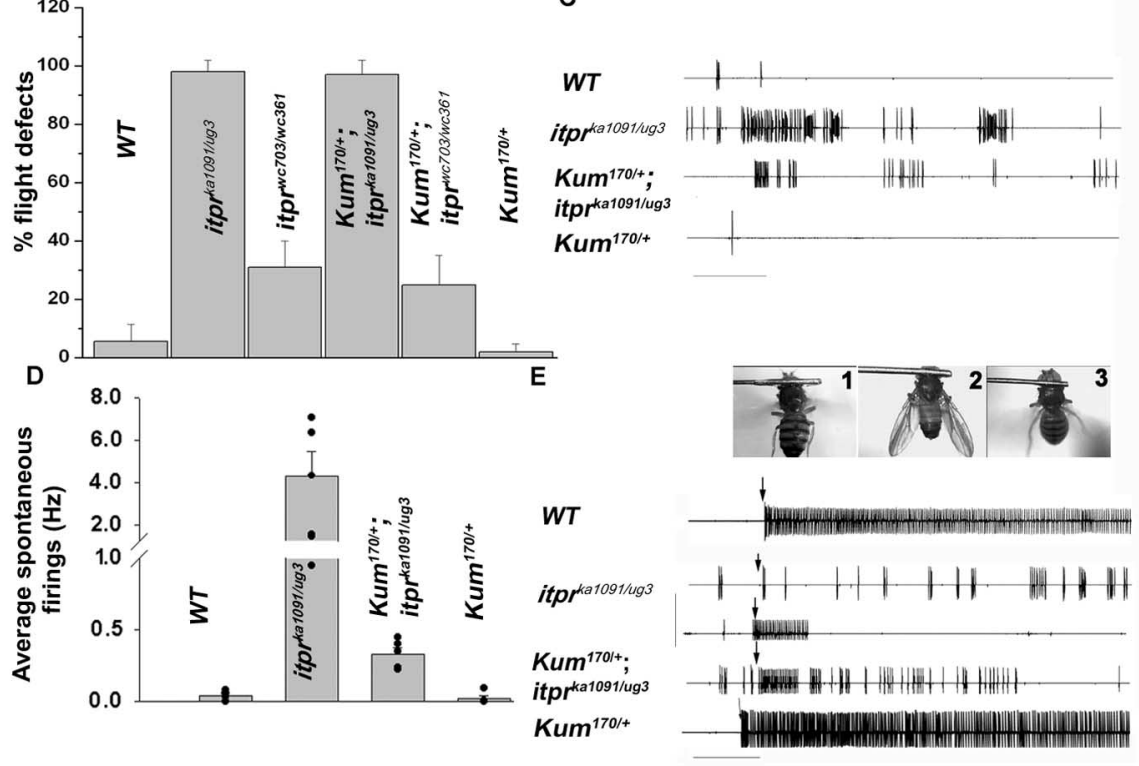

c) b)
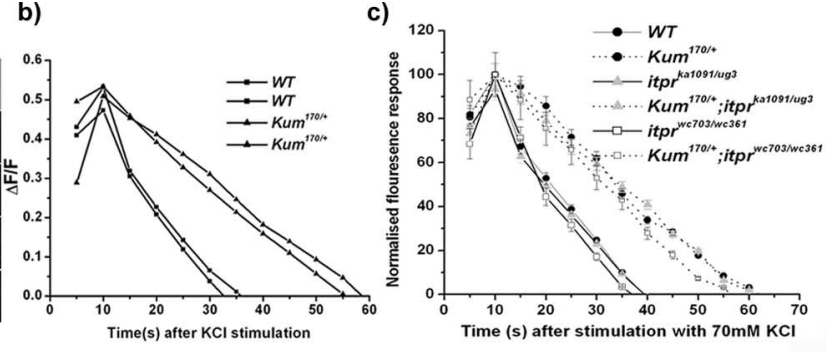

Figure 2. Effect of $\mathrm{Kum}^{170}$ on cytosolic $\mathrm{Ca}^{2+}$ and flight in itpr mutants. $\boldsymbol{A}, \mathrm{Kum}^{170}$ reduces the rate of $\mathrm{Ca}^{2+}$ clearance from the cytosol in primary larval neurons. $\boldsymbol{a}$, Time lapse images of larval neurons loaded with Fluo-3 from wild-type (WT) and Kum ${ }^{170 /+}$ animals before $(\boldsymbol{B})$ and after (see 5, 30,60 s) addition of $70 \mathrm{~mm} \mathrm{KCl}$ as a depolarizing stimulus. BF, Bright-field images; scale bar, 10 $\mu \mathrm{m} . \boldsymbol{b}$, Plots of the $\Delta F / F$ values based on the fluorescence signals recorded from individual neurons of the indicated genotypes. $\boldsymbol{C}$, Fluorescence levels $(\Delta F / F)$ normalized to the peak response (100\%) of the individual genotypes tested are shown. The solid lines indicate wild-type (filled circles), itpr $r^{\text {ka1091/ug3 }}$ (gray triangles), and $i \operatorname{tpr}^{\text {wc703/wc361 }}$ (open squares). The dashed lines indicate genotypes with one copy of $\mathrm{Kum}^{170}$ in wild-type, itpr ${ }^{\mathrm{ka} 1091 / u g 3}$, and itpr ${ }^{\mathrm{wC} 703 / \mathrm{wc} 361}$ organisms. In all six genotypes tested, larval neurons were loaded with Fluo-4. Peak values post-KCl depolarization were obtained from 45 or more individual neurons. The mean peak value $(\Delta F / F)$ for all six genotypes was similar $(p>0.01)$. $\Delta F / F$ values of strains with $K u m^{170}$ were significantly $(p<$ 0.05 ) different from the parent strain at all time points after the peak value. $\boldsymbol{B}$. Flight defects seen in itpr $r^{\text {ka1091/ug } 3}$ and $i_{\text {tpr }}^{\text {wc } 703 / \text { wC361 }^{2}}$ are not suppressed by Kum ${ }^{170 /+}$. Error bars denote SD. All itpr mutants and $\mathrm{Kum}^{170 /+}$; itpr mutants exhibit significant flight defects $(p<0.05)$ compared with wild-type (WT). Kum ${ }^{170 /+}$ organisms have normal flight behavior. $C$, Kum $^{170 /+}$ suppresses high levels of spontaneously generated action potentials. Calibration, 10 S. D, Quantification of spontaneously generated action potentials. The number of spikes observed in recordings over 2 min were counted and averaged from animals of the indicated genotypes to obtain individual firing frequencies in $\mathrm{Hz}$. Recordings were obtained from at least five animals of each genotype. Data are expressed as mean \pm SE. The spontaneous firing rate of $\mathrm{Kum}^{170 /+} ;$ itpr $^{\mathrm{ka} 1091 / \mathrm{ug} 3}$ organisms is significantly reduced $(p<0.05)$ when compared with itpr ${ }^{k a 1091 / u g 3}$. E, Air puff stimulated flight patterns in itpr $r^{k a 1091 / u g 3}$ and

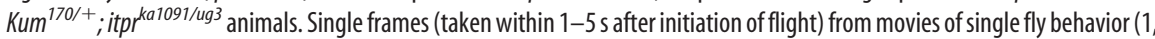
WT; 2, itpr $^{\text {ka1091/ug3 }} ; 3$, Kum $^{170 /+} ;$ itpr $^{\text {ka1097/ug3 }}$ ) are shown in the top panel (see also supplemental movie 1, available at wwW. jneurosci.org as supplemental material), whereas physiological recordings are shown in the bottom panel. On delivery of a gentle air puff, the DLMs of wild-type flies respond with a rhythmic pattern (wings are not visible for fly 1 in top panel because they are beating). This is absent in itpr ${ }^{\text {ka1091/ug } 3}$ flies in 10 of 10 animals tested (see stationary wings for fly 2). Kum ${ }^{170 /+}$; itpr ${ }^{\text {ka 1091/ug3 } 3}$ animals responded to an air puff by a rhythmic initiation phase (see fly 3 , in which wings are invisible because of wing beating). This generally terminates within $5 \mathrm{~s}$ of initiation. (Nine of 14 animals tested showed this initial rhythmic response.) This initial phase of response is accompanied by wing beating (see supplemental movie 1, available at www.jneurosci.org as supplemental material). The air puff response of $\mathrm{Kum}^{170 /+}$ flies is normal. The arrows mark the point of air puff delivery. Calibration, $5 \mathrm{~s}$.

\section{Drosophila $\mathrm{G} \alpha \mathrm{q}$ and PLC $\beta 21 \mathrm{C}$ mutants enhance InsP $\mathrm{P}_{3} \mathrm{R}$ phenotypes in larvae}

The differential suppression of itpr phenotypes by $\mathrm{Kum}^{170}$ led us to first investigate the mode of $\mathrm{InsP}_{3}$ receptor activation in Drosophila neurons in each phenotypic context. Ins $\mathrm{P}_{3}$ signaling in vertebrates can be initiated either by the activation of seven transmembrane domain receptors or receptor tyrosine kinases 
A
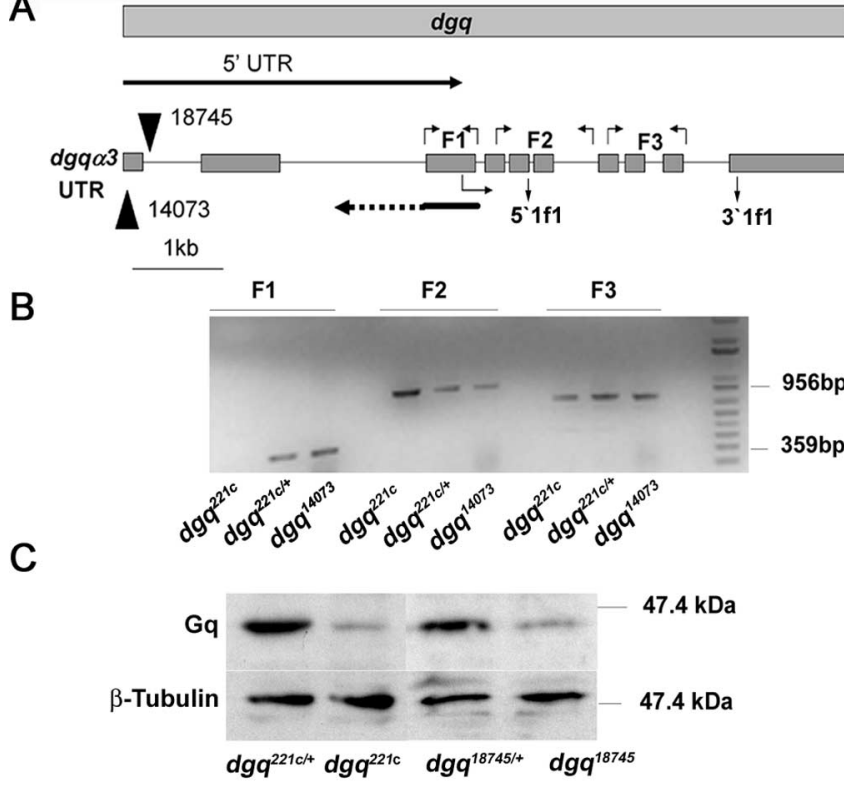

D

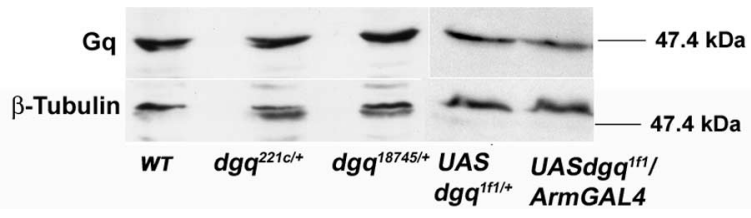

Figure 3. Characterization of mutant alleles for the $d g q$ locus. A, A schematic with the position of the P element (P14073) located in the $5^{\prime}$-UTR region of the dgq gene, which was used for generating the excision allele $d g q^{221 c}$. Also shown in this diagram is the position of $d g q^{18745}$ with corresponding exon/intron boundaries of the $d g q \alpha_{3}$ splice variant of the $d g q$ gene. F1, F2, and F3 denote different segments in the dgq gene that were selected for PCR amplification. Approximate positions of the primers used to generate UASdgq ${ }^{1 f 1}$ are shown as $5^{\prime} 1 \mathrm{f1}$ and $3^{\prime} 1 \mathrm{f1}$. B, PCRs performed on genomic DNA isolated from the indicated genotypes. The F1 fragment is absent in $d g q^{221 c}$ homozygous animals, whereas the $F 2$ and $F 3$ are present in all the genotypes tested. C, The $\operatorname{Dgq} \alpha 3$ protein is greatly reduced in larval homogenates of $d g q^{221 c}$ and $d g q^{18745}$ homozygous animals. A Western blot performed with homogenates of first-instar larvae of indicated genotypes shows a significant reduction of $\mathrm{Dgq} \alpha 3(\mathrm{Gq})$ protein in $d g q^{221 c}$ and $d g q^{18745}$ homozygous animals. D, No significant reduction in Dgq $\alpha 3(\mathrm{Gq})$ protein levels could be detected in $d g q^{221 c /+}$ and $d g q^{18745 /+}$ heterozygous animals compared with wild-type animals. A subtle reduction in $\mathrm{Gq} \alpha 3$ protein level was detected after ubiquitous expression of a dsRNA made against the dgq gene $\left(U A S d g q^{1 f 1}\right.$ ) compared with animals carrying a single copy of UASdgq $q^{1 f 1}$ insert.

(Berridge, 1993). The former class of receptors act via heterotrimeric G-proteins, consisting of an $\alpha$-subunit of the Gq class. In Drosophila, G $\alpha$ q is encoded by the dgq gene (Lee et al., 1990, 1994; Scott et al., 1995).

To test whether activation of the $\operatorname{Ins}_{3} \mathrm{R}$ occurs through $\mathrm{G} \alpha \mathrm{q}$, we obtained mutant $d g q$ alleles predicted to affect all known $d g q$ splice forms (Fig. 3). An existing mutant of $d g q\left(d g q^{393}\right)$ specifically affects splicing of the eye-specific splice variant $(d g q \alpha 1)$ and consequently the process of visual transduction (Scott et al., 1995). However, larval and adult brains express the $d g q \alpha 3$ splice variant, which is also present in other tissues (Ratnaparkhi et al., 2002). Based on its expression pattern, we predicted that mutant alleles affecting $d g q \alpha 3$ would be lethal. An excision allele of $d g q$ (221c) was generated by hopping out a P element (BL-14073) from the $5^{\prime}$ untranslated region (UTR) of $d g q$. The insertion site of P14073 lies $\sim 3.5 \mathrm{~kb}$ upstream of the translation start site of all Dgq isoforms (Fig. 3A) [details regarding the generation of $d g q^{221 c}$ will be published independently (P. Kain, G. Susinder, T. Senthil, V. Rodrigues, and G. Hasan, unpublished results)]. PCR
Table 1. Mutations in genes coding for components of the Ins $\mathrm{P}_{3}$ signaling pathway affect larval viability

\begin{tabular}{|c|c|c|c|}
\hline Genotypes & $80-88$ h AEL & $128-136 \mathrm{~h} \mathrm{AEL}$ & $320-328 \mathrm{~h} \mathrm{AEL}$ \\
\hline$i_{t p r}{ }^{w c 703 / w c 361}$ & $92.6 \pm 8.0$ & $98 \pm 2.0$ & $98.4 \pm 6.4$ \\
\hline$d g q^{221 c /+} ; i t p r^{w c 703 / w c 361}$ & $89.2 \pm 11.4$ & $94.6 \pm 2.4$ & $87.4 \pm 7.0$ \\
\hline plc21 ${ }^{P 319 /+} ;$ itpr wc703/wc361 $^{\text {wo }}$ & $98.4 \pm 1.2$ & $94.6 \pm 3$ & $92.6 \pm 5.8$ \\
\hline$d g q^{221 c} / p / c 21 c^{P 319} ; i t p r^{w c 703 / w c 361}$ & $72 \pm 3.4$ & $47.4 \pm 6.4$ & $34.0 \pm 4.0$ \\
\hline 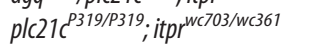 & $84.6 \pm 6.0$ & $5.4 \pm 2.4$ & Nil \\
\hline$s l^{1} / s l^{1} ; i t p r^{\mu g 3 / k a 1051}$ & $94.6 \pm 1.2$ & $96.6 \pm 3.0$ & $82.6 \pm 4.6$ \\
\hline$s P^{2} / s L^{2} ; i t p r^{\mu g 3 / k a 1091}$ & $96.0 \pm 5.2$ & $95.4 \pm 4.2$ & $82.0 \pm 5.2$ \\
\hline
\end{tabular}

Numbers represent percentage of survivors \pm SD.

analysis performed on genomic DNA obtained from $d g q^{221 c}$ homozygous larvae indicated the absence of a $359 \mathrm{bp}$ fragment in the region of the translation start site (Fig. $3 A, B$ ). This suggested that $d g q^{221 c}$ homozygotes would be devoid of zygotic Gq $\alpha$ protein. To verify this, the level of Dgq $\alpha 3$ was determined in larval lysates by carrying out Western blots in which Dgq $\alpha 3$ levels were detected with an antibody specific for this isoform (Ratnaparkhi et al., 2002). As expected, the intensity of the band corresponding to $\operatorname{Dgq} \alpha 3$ was considerably reduced in $d g q^{221 c}$ homozygotes on comparison with the level of another protein ( $\beta$-tubulin), and with the levels observed in $d g q^{221 c /+}$ heterozygotes (Fig. $3 C$ ). Dgq $\alpha 3$ levels are also reduced to a similar extent in a newly designated $d g q$ allele $\left(d g q^{18745}\right)$, obtained from the public stock center. The P-insert in this allele is in the first intron of $d g q$ as determined from the sequence of the neighboring genomic region, obtained from the BDGP (Fig. 3). We attribute the low level of $\operatorname{Dgq} \alpha 3$ seen in the mutant homozygotes to a maternal contribution. However, this has not been tested rigorously.

Animals homozygous for $d g q^{221 c}$ and $d g q^{18745}$ survive for $10-12 \mathrm{~h}$ posthatching, indicating an essential requirement of this gene in larvae (data not shown). A role for the itpr locus in larval neurons, in the context of viability, is known (Joshi et al., 2004). Consequently, we tested the effect of a single copy of $d g q^{221 c}$ on the viability of $i t p r^{w c 361 / w c 703}$ organisms. The viability profile of animals of the genotype $d g q^{221 c /+} ; i t r^{\text {wc703/wc361 }}$ appeared similar to that of $i t p r^{\text {wc703/wc361 }}$ (Table 1). To further reduce signaling through Gq $\alpha$ and PLC $\beta$, a single copy of an existing hypomorphic allele of plc21C [P319; (Weinkove et al., 1999)] was introduced to generate a strain of the genotype $d g q^{221 C} / p_{l c} 21 C^{P 319}$; $i t p r^{w c 703 / w c 361}$. Viability in this triple mutant strain was indeed reduced at all developmental stages tested, and only $40 \%$ of the animals survived until adulthood (Table 1). Thus, in the relatively broad context of larval viability, signaling through the Ins $\mathrm{P}_{3} \mathrm{R}$ is compromised by reducing levels of $\mathrm{G} \alpha \mathrm{q}$ and PLC $\beta$. The rationale of this experiment was based on biochemical and pharmacological studies from vertebrates in which it is known that activation of Gq $\alpha$ leads to stimulation of the enzyme PLC $\beta$, which cleaves phosphatidylinositol 1,4-bisphosphate $\left(\mathrm{PIP}_{2}\right)$ to generate Ins $\mathrm{P}_{3}$. The Drosophila genome has two genes coding for PLC $\beta$, referred to as plc21C and norpA. Among these, plc21C is expressed ubiquitously (Shortridge et al., 1991), whereas norpA is expressed strongly in the eyes and is essential for visual transduction (Bloomquist et al., 1988). The effect of norpA mutant alleles on the viability of itpr mutants is currently under investigation.

In vertebrates, cleavage of PIP $_{2}$ by PLC $\gamma$ has been shown to occur after activation of receptor tyrosine kinases (Rebecchi and Pentyala, 2000). In Drosophila, a single gene with homology to PLC $\gamma$ has been described (Thackeray et al., 1998). This form of PLC $\gamma$ shares an equal degree of similarity with two forms of PLC $\gamma$ found in mammals (Manning et al., 2003). We tested whether 
A

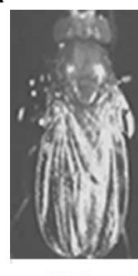

WT

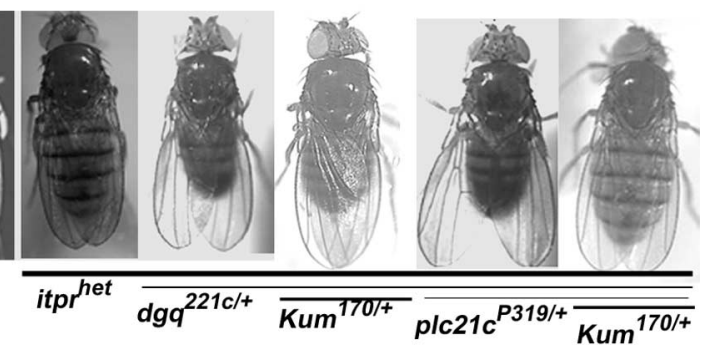

B

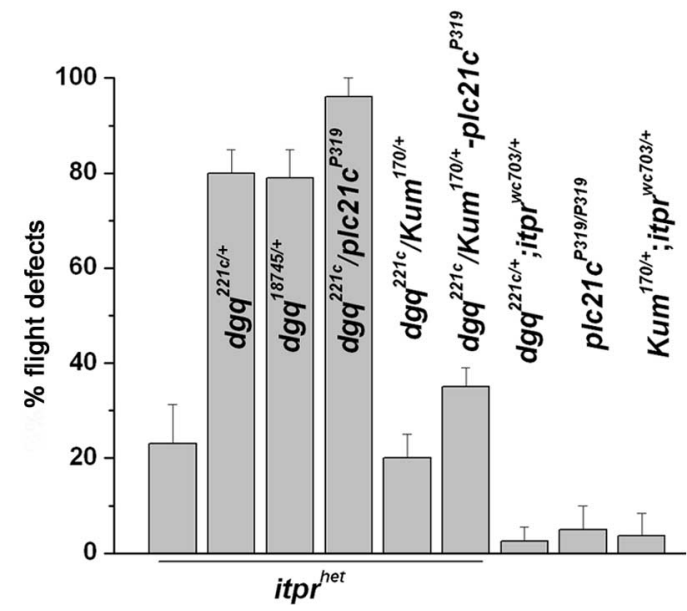

Figure 4. A balance of Ins $\mathrm{P}_{3}$ signaling and SERCA activity controls wing posture and flight behavior. A, Maintenance of wing posture. Normal wing posture in itpr het $\left(i t p r^{\text {wc361/wc703 }}\right.$ animals is lost after introducing a single copy of $d g q^{221 c}$ leading to a mild defect $\left(d g q^{221 c /+}\right.$; $i_{p r}{ }^{w c 703 / w c 361}$ ). Defective wing posture is further enhanced in animals with mutations in three genes of the $\mathrm{Ins}_{3}$ signaling pathway $\left(\mathrm{dgq} \mathrm{q}^{221 \mathrm{c}} / \mathrm{plc} 21 \mathrm{c}^{P 319} ;\right.$ itpr $\left.^{\text {wc703/361 }}\right)$. Defective wing posture in these animals is suppressed by introduction of the $\mathrm{Kum}^{170}$ allele. $\boldsymbol{B}$, Flight behavior. Flies with a single copy of $d g q^{221 c}$ in the background of itpr het $\left(\mathrm{dgq}^{221 / 1+} ;\right.$ itpr $^{\text {wc703/wc361 }}$ and $d g q^{18745 /+}$; $i_{\text {itpr }}^{\text {wc703/wc361 }}$ ) exhibit severe flight defects ( 85\%) compared with itpr ${ }^{\text {wc703/wc361 }}(\sim 30 \%)$ $(p<0.001)$. This defect was further enhanced by introducing a single copy of $p / c 21 c^{p 319}$ $\left(\mathrm{dgq} q^{221 c} / \mathrm{plc} 21 \mathrm{c}^{P 319} ; i_{\mathrm{tpr}} \mathrm{wc}^{\mathrm{W} 703 / \mathrm{wc} 361}\right)(\sim 98 \%)(p<0.001)$. Enhanced flight defects arising from introduction of $d g q^{221 c}$, plc $21 c^{P 319}$ mutant alleles $\left(d g q^{221 c /+} ; i \operatorname{tpr} r^{w c 703 / w c 361}\right.$ and $d g q^{221 c} /$ plc21 $c^{P 319} ;$ itpr $^{\text {wc703/wc361 }}$ ) were suppressed by $\mathrm{Kum}^{170}$ and returned to the level seen in $i_{p r}{ }^{w c 703 / w c 361}(p<0.05)$. Error bars indicate SEM.

mutant alleles in the PLC $\gamma$ gene (small wing $-s l^{1} ; s l^{2}$ ) enhanced larval lethality in itpr mutants. Both $s l$ mutant alleles have been designated as null (Thackeray et al., 1998). We monitored the survival of $s l^{l}$; itpr ${ }^{k a 1091 / u g 3}$ or $s l^{2}$; itpr ${ }^{k a 1091 / u g 3}$ males at different stages of development. The survival profile of $s l$; itpr double mutant animals is similar to animals carrying mutations in the $i t p r$ locus alone (Table 1). Moreover, adult phenotypes described for the $s l$ mutants were not enhanced (data not shown). Thus, Drosophila PLC $\gamma$ appears to play no discernable role in the activation of the InsP $\mathrm{P}_{3} \mathrm{R}$. This finding is consistent with previously described phenotypes of $s l$ mutants, none of which overlaps with phenotypes described for adult viable itpr mutants (Thackeray et al., 1998; Manning et al., 2003; Joshi et al., 2004).

\section{Wing posture and flight behavior defects in itpr mutants are} enhanced by a single copy of $d g q$ mutant alleles

The newly characterized $d g q$ mutant alleles were tested for their effect on flight phenotypes associated with itpr mutants. For this purpose, the itpr mutant combination of $i t p r^{w c 703 / w c 361}$ was chosen. On introduction of a single copy of $d g q^{221 c}$ in $i t p r^{w c 703 / w c 361}$ organisms, a slight change in wing posture was evident. This was further enhanced in flies of the genotype $d g q^{221 c} / p l c 21 c^{P 319}$; $i t p r^{w c 703 / w c 361}$ (Fig. 4). Similar results were obtained with $d g q^{18745}$ (data not shown). Quantification of the wing posture seen in these genotypes was not possible primarily because of the fact that wing posture undergoes alterations during anesthetization in wild-type and mutant flies.

To study the effect of $d g q$ mutant alleles on flight behavior, flies of different genotypes were tested in the cylinder drop test. The 30\% flight defects seen in $i t p r^{w c 703 / w c 361}$ animals were enhanced to $80 \%$ by introducing a single copy of either $d g q^{221 c}$ or $d g q^{18745}$ (Fig. 4B). Next, we assessed the effect of mutant alleles of plc21c and norpA on flight behavior of $d g q$ and itpr mutant combinations. As expected, from the altered wing posture of the $d g q^{221 c} / p l c 21 c^{P 319}$; $i t p r^{w c 703 / w c 361}$ flies (Fig. 4A), these organisms exhibit poor flight ( $\sim 95 \%$ are flight defective) (Fig. $4 B$ ). In comparison, flight defects in males of the genotype norp $A^{P 24}$; $d g q^{221 c} /+$; $i t p r^{w c 703 / w c 361}$ were no different from $d g q^{221 c} /+$; $i \operatorname{tpr}^{w c 703 / w c 361}$ (76 \pm 8 and $80 \pm 5 \%$, respectively). None of the PLC $\beta$ mutant strains has flight defects by itself (Fig. $4 B$ ) (data not shown). Furthermore, the two mutant alleles tested, $p l c \beta 21 C^{P 319}$ and norp $A^{P 24}$, did not enhance flight defects in itpric703/wc361 organisms (data not shown). Because $p l c \beta 21 C^{P 319}$ is a viable, weak hypomorph, its effect is presumably evident only in a sensitized genetic background that includes $d g q$ and $i t p r$ mutant alleles. In contrast, norpA ${ }^{P 24}$ is a null allele (Pearn et al., 1996). The absence of a genetic interaction between this allele and either $i t p r^{w c 703 / w c 361}$ or $d g q^{221 c} /+; i t p r^{w c 703 / w c 361}$ suggests that there is a nonredundant role for the plc21c gene product in adult wing posture and flight behavior.

\section{Effects of $d g q$ and plc21c mutants on flight physiology}

The enhancement of flight behavior defects in $d g q^{221 c} / p l c 21 c^{P 319}$; $i t p r^{w c 703 / w c 361}$ animals could arise from either the enhanced wing posture defect or a change in the neural circuits underlying flight behavior or both. To distinguish between these possibilities, the patterns of spontaneous firing and air puff-induced flight were recorded from the DLMs of flies of the genotype $i$ tpr $^{\text {wc703/wc361 }}$ and $d g q^{221 c /+} ; i t p r^{w c 703 / w c 361}$. As shown in Figure $5, A$ and $B$, the spontaneous firing rate recorded from the DLMs of $i t p r^{w c 703 / w c 361}$ flies is no different from that of wild-type flies. In contrast, the DLMs of $d g q^{221 c /+}$; itpr $r^{w c 703 / w c 361}$ animals exhibit mild endogenous hyperactivity (Fig. $5 A, B$ ). Interestingly, the rate of spontaneous firing was altered only minimally in $d g q^{18745 /+} ; i t p r^{\text {wc } 703 / w c 361}$ animals (Fig. 5), whereas animals of the genotype $d g q^{221 c}$ / plc21 $c^{P 319} ;$ itpr ${ }^{w c 703 / w c 361}$ showed no additional increase in their spontaneous firing rate compared with $d g q^{221 c /+} ; i t p r^{w c 703 / w c 361}$ animals (data not shown). These observations are consistent with previous results in which altered wing posture and increased spontaneous firing could be rescued independently (Banerjee et al., 2004). Next, all organisms were tested in the cylinder drop test and separated into two classes referred to as "fliers" and "nonfliers," based on their performance in the test. The "flier" population of $i t p r^{w c 703 / w c 361}$ flies responded to an air puff with a rhythmic train of action potentials similar to that observed in wild-type flies. This rhythmic response lasts for $30 \mathrm{~s}$ or more. Nonfliers of the same genotype exhibit an initial rhythmic response after the air puff, but fail to maintain the response after $5 \mathrm{~s}$ or less (Fig. $5 C$ ). Nearly identical patterns of response were obtained from nonfliers of the genotypes $d g q^{221 c} /+$; $i t p r^{w c 703 / w c 361}$ and $d g q^{18745} /+$; $i t p r^{w c 703 / w c 361}$. Moreover, the response from DLMs of triple mutant animals $\left(d g q^{221 c} / p l c 21 c^{P 319} ; i t p r^{w c 703 / w c 361}\right)$ was also no differ-

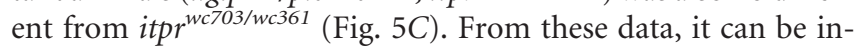
ferred that the enhanced flight defects in $d g q$, itpr double mutants and $d g q$, plc21C, itpr triple mutants arise from a combination of 

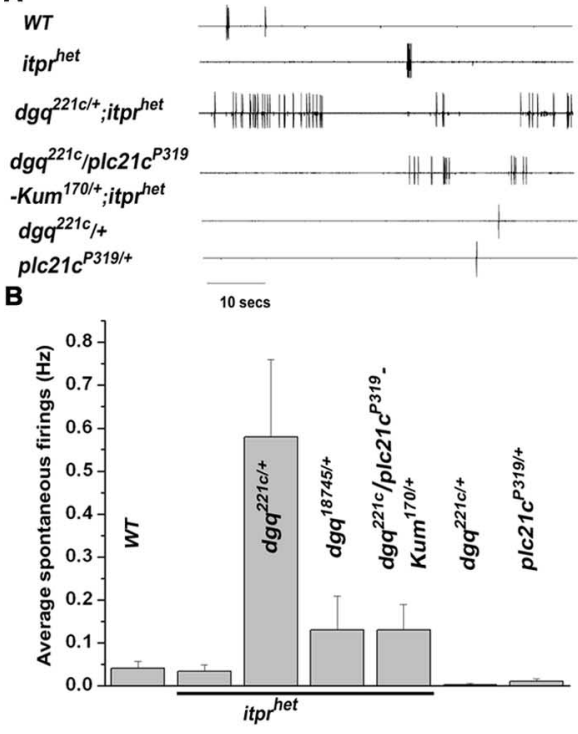

$c$
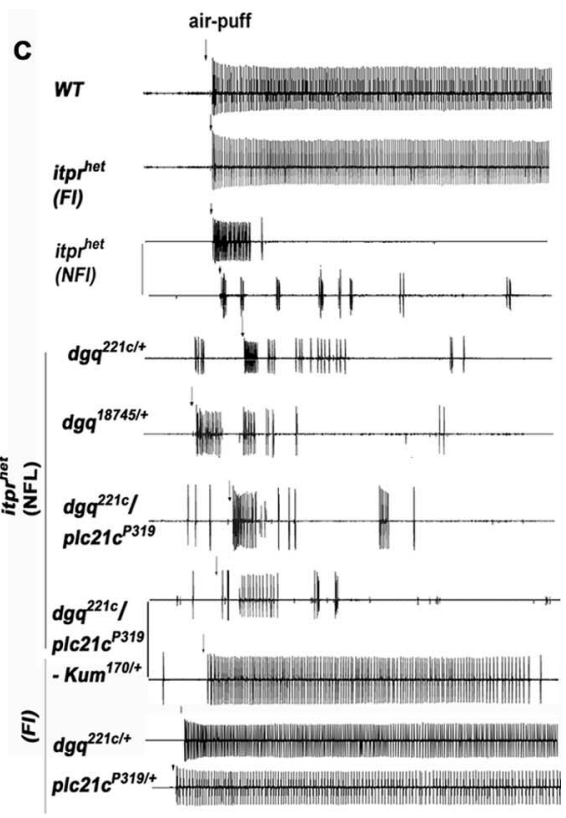

$5 \operatorname{secs}$

Figure 5. $\mathrm{Gq} \alpha$ and SERCA mutants affect the rate of spontaneous firing in weak $\operatorname{lns} \mathrm{P}_{3} \mathrm{R}$ mutants but have no effect on flight patterns. A, A low level of spontaneous firing was recorded from DLMs of wild-type and $i t p r^{w C 703 / w c 361}$ (itpret) organisms.

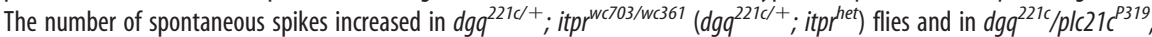
$i_{\text {tpr }}{ }^{w C 703 / w c 361}$ flies (data not shown). This increase in spontaneous firing was suppressed by the introduction of Kum ${ }^{170 /+}$

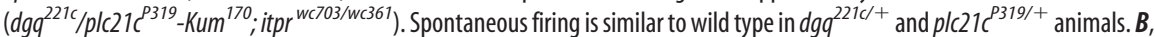
Average numbers of spikes were calculated as described in Figure 2. Error bars indicate SEM. A significantly enhanced rate of firing was observed in $d g q^{221 / 1+} ; i t p r^{\text {wC703/wc361 }}$ animals compared with itpr ${ }^{\text {wc703/wc361 }}$ animals $(p<0.05)$. Introduction of Kum ${ }^{170}$

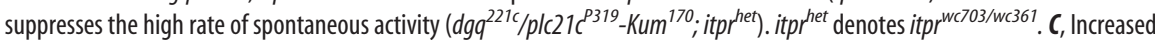
penetrance of flies with defective flight patterns in dgq,itpr double and dgq,plc21c,itpr triple mutants. Air puff induced flight in wild-type flies (WT). The rhythmic firing of action potentials measured from DLMs of WT continues throughout the duration of flight. A majority of the flight-competent animals of $\mathrm{itpr}^{\mathrm{wc} 703 / \mathrm{wc} 361}$ ( $\mathrm{Fl}$, selected through the flight column assay) genotype (4 of 5) have flight patterns similar to WT animals. Nonflier animals (NFI) of itpr ${ }^{\text {wc703/367 }}$ genotype exhibit an initial response to the puff of air but usually fail to sustain the response (4 of 7). The penetrance of this defective flight pattern was higher in $d g q^{221 c /+}$;

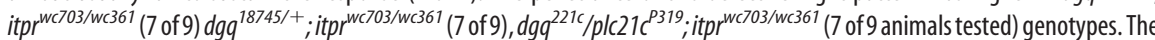

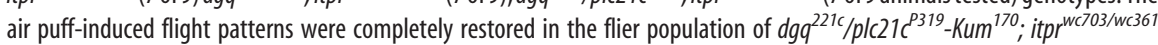
genotype ( 3 of 3 ), whereas in the nonfliers of this genotype flight patterns remained defective ( 4 of 5 ). Flight patterns in $d g q^{221 c /+}$ and $p / c 21 c^{P 319 /+}$ animals are like WT. Calibration, $5 \mathrm{~s}$.

the altered wing posture and changes in the neural circuit underlying flight.

\section{$\mathrm{Kum}^{170}$ can suppress phenotypes enhanced by $d g q$ and $p l c 21 \mathrm{C}$ in itpr mutants}

The results obtained so far show that itpr mutant phenotypes of altered wing posture and increased spontaneous firing from the DLMs can be suppressed by reducing SERCA activity and enhanced by reducing levels of Dgq $\alpha$ and PLC21C. These observations are consistent with the idea that the observed enhancement and suppression arise from opposing effects on the cellular output of $\mathrm{InsP}_{3}$ signaling viz. $\mathrm{Ca}^{2+}$ release from ER stores. To test this idea more rigorously, we asked whether the $\mathrm{Kum}^{170}$ mutation could suppress increased spontaneous firing from DLMs, wing posture, and flight defects in $d g q^{221 c /+} ; i t p r^{w c 703 / w c 361}$ and $d g q^{221 c /}$ plc21 $c^{P 319} ; i t p r^{w C 703 / w c 361}$ animals. As shown in Figure 4, the $\mathrm{Kum}^{170}$ mutation in SERCA could suppress wing posture and flight defects in $d g q$; itpr double mutants $\left(\mathrm{dgq}^{221 \mathrm{c}} / \mathrm{Kum}^{170}\right.$; $\left.i t p r^{w c 703 / w c 361}\right)$ and in $d g q / p l c 21 c$; itpr triple mutants $\left(d g q^{221 c} /\right.$ plc21 $c^{P 319}-\mathrm{Kum}^{170} ; \mathrm{itpr}^{\text {wc703/wc361 }}$ ). Furthermore, the enhanced spontaneous firing recorded from the DLMs of $d g q^{221 c /+}$; $i t p r^{w c 703 / w c 361}$ flies was also suppressed in $d g q^{221 c} / \mathrm{Kum}^{170}$; $i t p r^{w c 703 / w c 361}$ and $d g q^{221 c} / p l c 21 c^{P 319}-K^{2} m^{170}$; itpr $r^{2 c 703 / w c 361}$ flies

(Fig. 5A,B). In all genotypes tested, flight patterns in response to a gentle air puff seen in nonflier animals were comparable with that of flight-defective $i t p r^{w C 703 / w c 361}$ animals (Fig. 5C).

\section{Tissue specificity of G $\alpha q-\mathrm{InsP}_{3}$ receptor signaling}

Although the data are consistent with a model in which all mutants act within the same cell (or cells) to eventually result in the observed phenotypes, given the pleiotropic nature of calcium signaling, the observed genetic interactions could arise from differential effects in multiple cell types. In a previous report, we showed that defective flight behavior and related phenotypes in itpr mutants can be rescued by expression of a wild-type $i t p r^{+} \mathrm{cDNA}$ in aminergic interneurons (Banerjee et al., 2004). To test the cellular of focus of $d g q$ enhancement of itpr mutant flight defects, a UASdsRNA construct was generated against the $d g q$ gene (UASdgq $q^{l f 1}$ ) (for details of the construct, see Materials and Methods). Ubiquitous expression of this construct with ArmGAL4 results in marginally lower levels of Dgq $\alpha 3$ in larvae (Fig. 3D). The UASdgq ${ }^{1 f 1}$ transgene was turned on specifically in aminergic neurons of $i t p r^{w c 703 / w c 361}$ organisms with the Dopa-decarboxylase GAL4 strain (DdcGAL4), which drives expression in all aminergic cells and neurons in Drosophila (Li et al., 2000). A significantly greater percentage of these flies are flightless $(60 \%)$, compared with $i t p r^{w c 703 / w c 361}$ (30\%) (Fig. 6B). However, flies expressing $U A S d g q^{I f 1}$ in aminergic neurons do not show any change in wing posture and the rate of spontaneous firing (data not shown). Moreover, the introduction of UASdgq $q^{1 f 1}$ does not alter the air puff response of $i t p r^{w c 703 / w c 361}$ (compare Figs. 6D, 5C). These data suggest that the enhanced flight defects in dqq;itpr double mutants are primarily a consequence of reducing Dgq $\alpha 3$ levels in the DdcGAL4 domain, but the reduction in Dgq $\alpha 3$ is probably less than that obtained with a single copy of $d g q$ mutant alleles. The absence of flight phenotypes in DdcGAL4/UASdgq ${ }^{l f 1}$ organisms (Fig. $6 \mathrm{~B}$ ) is consistent with the observation that this transgene lowers Dgq $\alpha 3$ levels to a modest extent only (Fig. 3D). It also agrees with the phenotypes of $d g q^{221 c /+}$ and $d g q^{18745 /+}$ organisms, which exhibit normal flight behavior yet are able to enhance itpr mutant phenotypes in single copy (Fig. 4) (data not shown).

In an alternate approach, cell and tissue specificity of Gq $\alpha$ and $\mathrm{InsP}_{3}$ signaling was addressed by expression of an UASdgq $\alpha 3^{+}$ transgene in the DdcGAL4 domain of $d g q^{221 c /+} ; i t p r^{w c 703 / w c 361}$ animals (Fig. 6). In DdcGAL4-dgq $q^{221 c} / U A S d g q \alpha 3^{+}$; $i t p r^{w c 703 / w c 361}$ animals, wing posture was rescued partially (Fig. $6 \mathrm{~A}$ ). The wingrescued flies were selected visually and tested for flight in the flight column, in which $\sim 45 \%$ passed the flight test compared with $15 \%$ of $d g q^{221 c /+}: i t p r^{w c 703 / w c 361}$ animals (Fig. 6 B). The rescued animals also showed a reduction in spontaneous firing (Fig. $6 C)$. Flight patterns, in response to an air puff, were restored in 


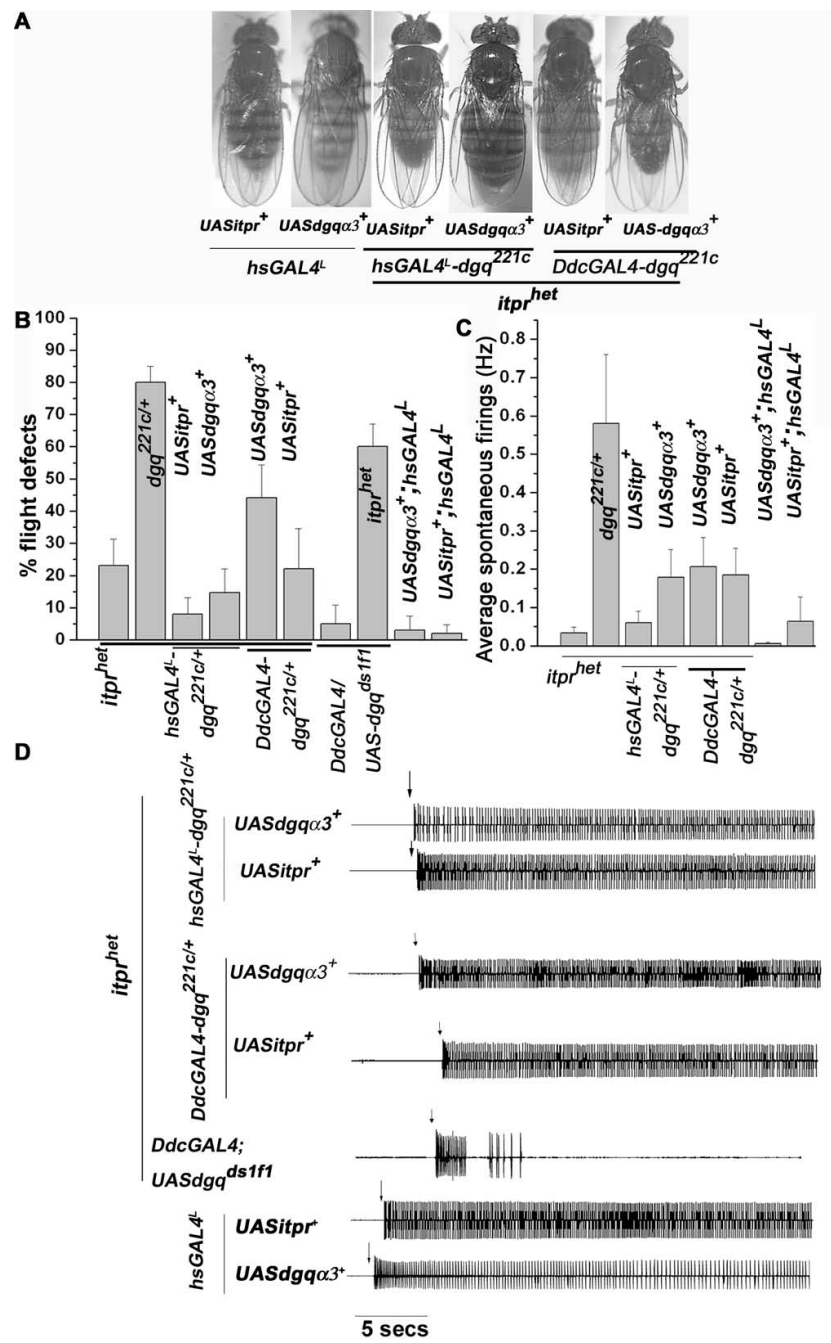

Figure 6. The cellular focus of $\mathrm{Gq}-\operatorname{Ins} \mathrm{P}_{3} \mathrm{R}$ interactions leading to loss of flight and neuronal rhythmicity lies in aminergic interneurons. $\boldsymbol{A}$, Rescue of wing posture defects in $\mathrm{Gq}-\mathrm{Ins} \mathrm{P}_{3} \mathrm{R}$ mutants. Ubiquitous expression of either UASdgq $\alpha 3^{+}$or UASitpr ${ }^{+}$in dgq, itpr double-mutant animals $\left(\mathrm{dgq}^{221 \mathrm{c} /+} ; \mathrm{itpr}^{\mathrm{wc} 703 / \mathrm{wc} 361}\right)$ showed rescue of wing posture. The rescue was also seen when $\mathrm{UASitpr}^{+}$or $\mathrm{UASdgq} 3^{+}$expression was confined to the aminergic domain. $\boldsymbol{B}$, Manipulating levels of Ins $\mathrm{P}_{3}$ signaling in aminergic neurons modulates flight behavior. Expression of UASdgq $\alpha 3^{+}$or UASitpr ${ }^{+}$either in aminergic neurons (DdcGAL4) or ubiquitously (hSGAL4 ${ }^{L}$ ) suppresses flight defects seen in $d g q^{221 / /+}$; itpr ${ }^{w c 703 / w c 361}$ animals $(p<0.05)$. Alternately, expression of a dsRNA construct for dgq $\left(\right.$ UASdgq $^{171}$ ) in aminergic neurons of itpr ${ }^{\mathrm{wC} 703 / \mathrm{wc} 361}$ (DdcGAL4/UASdgq $q^{171} ;$;itpr ${ }^{\text {wC703/wC361 }}$ ) animals results in increased flight defects compared with itpr $^{\text {wc703/wC361 }}$ or DdcGAL4/UASdgq $q^{1 f 1}$ animals ( $p<0.05$ for both genotypes). Ubiquitous expression of UASdgq $\alpha^{3}{ }^{+}$or UASitpr ${ }^{+}$has no effect on flight behavior. C, Cell type-specific suppression of neuronal hyperactivity. Flies ubiquitously expressing either UASdgq $\alpha 3^{+}$

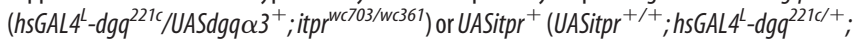
itpr ${ }^{\text {wc } 703 / w C 361}$ ) show low levels of spontaneous activity compared with $d g q^{221 c /+}$; itpr rc $^{\text {rc } 03 / w C 361}$ animals $(p<0.05)$. Expression of either UASdgq $\alpha 3^{+}$or UASitpr ${ }^{+}$in aminergic neurons is sufficient to suppress neuronal hyperactivity seen in flies with compromised InsP

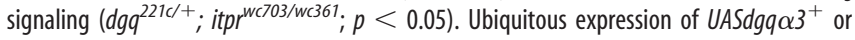
$\mathrm{UASitpr}^{+}$has no effect on spontaneous firing. Quantification of average spontaneous firing rates was done as described in Figure 2 and Materials and Methods. Error bars indicate SEM. D, Restoration of flight patterns in response to an air puff. Flight-competent flies were selected after flight test experiments presented in $\boldsymbol{B}$ and subjected to an air puff. Normal flight patterns were restored in response to

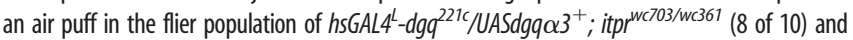

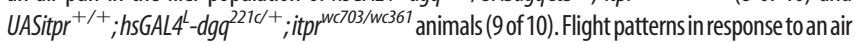

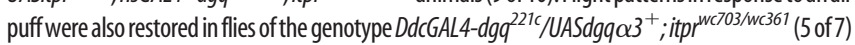

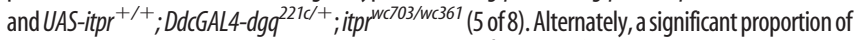
flight-defective flies of the genotype DdcGAL4/UASdgq ${ }^{171} ;$ itpr ${ }^{\mathrm{wC} 703 / \mathrm{wc} 361}$ showed air puff response defects ( 4 of 7). Expression of UASitpr ${ }^{+/+}$( 3 of 3 ) or UASdgq $\alpha 3^{+}$( 6 of 6 ) ubiquitously with the help of hSGAL4L did not affect air puff response. the majority of fliers of the genotype DdcGAL4- $d q q^{221 c} / U A S$ $d g q \alpha 3^{+}$; $i t p r^{w c 703 / w c 361}$ (Fig. 6D), similar to what is observed in fliers from $i t p r^{w c 703 / w c 361}$ (Fig. 5B). As controls, wing posture, flight, and related responses were measured in a number of related genotypes (Fig. 6). Interestingly, the expression of UAS$d g q \alpha 3^{+}$in the DdcGAL4 domain resulted in a slightly defective wing posture (data not shown) suggesting that overexpression of Dgq $\alpha 3$ in DdcGAL4 cells can have deleterious effects. This observation also provides a possible explanation for why UASdgq $\alpha 3^{+}$ rescue of flight in $d g q^{221 c} /+$; itpr $r^{w c 361 / w c 703}$ organisms is poor compared with rescue by the UASitpr ${ }^{+}$transgene. Ubiquitous expression to a low level by the hsGAL4 ${ }^{\mathrm{L}}$ driver at $25^{\circ} \mathrm{C}$ results in similar levels of rescue of flight by both transgenes (Fig. 6).

\section{Discussion}

Intracellular $\mathrm{Ca}^{2+}$ release through the $\operatorname{Ins}_{3} \mathrm{R}$ is a feature of all multicellular organisms in which it is thought to shape the temporal and spatial aspects of calcium signaling in both excitable and nonexcitable cells (Berridge et al., 2003). The results presented here demonstrate that calcium signals generated through the $\mathrm{InsP}_{3} \mathrm{R}$ can have distinct attributes that lead to different phenotypes at the systemic level. First, the InsP ${ }_{3} \mathrm{R}$ elevates $\left[\mathrm{Ca}^{2+}\right]_{\mathrm{i}}$ concentration in a manner that can be compensated in $\operatorname{InsP}_{3} \mathrm{R}$ mutants by decreasing the rate of calcium reentry back into the ER store. Second, it generates a class of $\mathrm{Ca}^{2+}$ signals that appear to depend on the intrinsic properties of the $\operatorname{Ins}_{3} R$. These are neither suppressed by slower entry of $\mathrm{Ca}^{2+}$ into the ER nor enhanced by reducing the activity of components that lie upstream of the InsP $\mathrm{P}_{3} \mathrm{R}$ (supplemental Fig. 1, available at www.jneurosci. org as supplemental material). The precise nature of these two classes of calcium signals needs to be elucidated further. Intuitively, the two might activate separate sets of signaling molecules in the cytosol. For example, the information in a given $\mathrm{Ca}^{2+}$ signal derives both from the amplitude of $\mathrm{Ca}^{2+}$ elevation and from the frequency of $\mathrm{Ca}^{2+}$ oscillations (Berridge, 1993; Thorn et al., 1993a,b) that ensue. Although amplitude and frequency based signals can both be decoded by transcription factors (Dolmetsch et al., 1997, 1998) with differential sensitivity to $\mathrm{Ca}^{2+}$, oscillatory $\mathrm{Ca}^{2+}$ signals are decoded by cytosolic effectors like CaMKII $\left(\mathrm{Ca}^{2+} /\right.$ calmodulin-dependent protein kinase II) (De Koninck and Schulman, 1998). At a cellular level, oscillatory signals are known to control secretion (Hille et al., 1994) and neuronal differentiation (Spitzer et al., 2000). Our study shows that different $\mathrm{Ca}^{2+}$ signals generated through the Ins $\mathrm{P}_{3} \mathrm{R}$ act at multiple levels to finally control systemic phenotypes such as flight behavior.

\section{Interaction between the InsP $\mathrm{P}_{3} \mathrm{R}$ and SERCA}

An interaction between the InsP $\mathrm{P}_{3} \mathrm{R}$ and SERCA is not unexpected but has not been demonstrated to our knowledge in vivo. Pharmacological blockage of the SERCA pump is known to cause store depletion and a gradual elevation of cytosolic $\mathrm{Ca}^{2+}$ because of an unopposed $\mathrm{Ca}^{2+}$ leak from intracellular $\mathrm{Ca}^{2+}$ stores (Thastrup et al., 1990; Mason et al., 1991). Although complete depletion of store $\mathrm{Ca}^{2+}$ would abolish intracellular $\mathrm{Ca}^{2+}$ release through the $\mathrm{InsP}_{3} \mathrm{R}$ on agonist stimulation, a partial blockage of SERCA activity may result in slower uptake of released $\mathrm{Ca}^{2+}$, leading to modest but prolonged elevation of $\left[\mathrm{Ca}^{2+}\right]_{\mathrm{i}}$. The fact that $\mathrm{Kum}^{170}$ suppresses rather than enhances $\mathrm{InsP}_{3} \mathrm{R}$ phenotypes suggests that this mutant allele (at the temperatures tested) slows down calcium uptake into ER stores after agonist stimulation. Slower return to basal $\left[\mathrm{Ca}^{2+}\right]_{\mathrm{i}}$ after $\mathrm{KCl}$ depolarization supports this idea. It is possible that $\mathrm{Kum}^{170}$ also lowers luminal $\mathrm{Ca}^{2+}$ content in the ER. We have not directly measured the luminal 
$\mathrm{Ca}^{2+}$ concentration in $\mathrm{Kum}^{170 /+}$ animals. However, because $\mathrm{Kum}^{170}$ did not enhance any of the described itpr phenotypes, it seems unlikely that luminal $\mathrm{Ca}^{2+}$ concentrations in this strain have fallen to a level that negatively impacts $\mathrm{Ins}_{3} \mathrm{R}$ function.

The reduced rate of $\mathrm{Ca}^{2+}$ uptake into the ER could compensate for reduced $\operatorname{Ins}_{3} \mathrm{R}$ function by different mechanisms. The simplest explanation, which we favor, is that slower $\mathrm{Ca}^{2+}$ uptake from the cytosol attributable to $\mathrm{Kum}^{170}$ compensates directly for attenuated $\mathrm{Ca}^{2+}$ release in the neurons of $i t p r^{k a 1091 / u g 3}$ animals. This would be similar to the priming of oxytocin release on application of thapsigargin in the dendritic terminals of oxytocin neurons (Ludwig et al., 2002). However, at this stage, our results do not show unambiguously that the suppressor effect of $\mathrm{Kum}^{170}$ in itpr mutants is within the same cell or cells. A transgene encoding mutant $\mathrm{Kum}^{170}$ is available and experiments with this are in progress. Clearly, although reduced, SERCA activity in $\mathrm{Kum}^{170 /+}$ animals can suppress several defects in itpr mutants including larval viability and certain aspects of development of the air puffinduced flight circuit responsible for wing posture, neuronal hyperactivity, and flight initiation. Our data suggest that, for the cellular processes underlying these phenotypes, maintaining a certain cytosolic level of $\left[\mathrm{Ca}^{2+}\right]_{\mathrm{i}}$ on agonist stimulation is sufficient. Precisely which cellular process(es) is affected in itpr mutants remains unclear at this stage. It could include dendritic remodeling during pupal development (Duch and Levine, 2002), targeting of growth cones as observed in vitro in Xenopus (for review, see Gomez and Zheng, 2006) and the specification of neurotransmitters and their receptors during development of $\mathrm{Xe}$ nopus (Borodinsky et al., 2004).

The failure of $\mathrm{Kum}^{170}$ to sustain a rhythmic train of action potentials (necessary for a normal flight response) suggests that this phenotype does not depend solely on an increase in cytosolic $\left[\mathrm{Ca}^{2+}\right]_{\mathrm{i}}$ on $\mathrm{InsP}_{3}$-mediated $\mathrm{Ca}^{2+}$ release. Possibly sustained air puff-induced flight patterns are dependent on the intrinsic properties of the $\mathrm{InsP}_{3}$ receptor (as discussed further below).

Interestingly, the interplay of SERCA and $\mathrm{Ins}_{3} \mathrm{R}$ goes beyond the phenotypes described here. In vertebrate models of two neurodegenerative conditions, spinocerebellar ataxia (SPA) and $\mathrm{Ni}$ emann-Pick A disease, a downregulation of $\operatorname{InsP}_{3} \mathrm{R}$ and SERCA is observed well before the manifestation of disease symptoms (Lin et al., 2000; Ginzburg and Futerman, 2005). Both diseases are characterized by the selective loss of Purkinje neurons in the cerebellum known to express high levels of $\operatorname{Ins}_{3} \mathrm{R}$ and SERCA. Several other proteins implicated in $\mathrm{Ca}^{2+}$ signaling remain unaffected in SPA (Lin et al., 2000). These data suggest the existence of a widespread mechanism for $\mathrm{Ca}^{2+}$ signaling in neurons, which requires both $\mathrm{InsP}_{3}$-mediated $\mathrm{Ca}^{2+}$ release and SERCA activity. Altering this might lead to neurodegeneration. The Drosophila model of altered flight behavior thus opens up a new avenue of investigating how these two proteins together control cellular $\mathrm{Ca}^{2+}$ levels and downstream $\mathrm{Ca}^{2+}$-dependent processes. The effect of itpr mutants on $\mathrm{Ca}-\mathrm{P} 60 \mathrm{~A}$ levels and vice versa is currently under investigation.

\section{The Gq-PLC $\beta$ pathway regulates $\mathrm{InsP}_{3}$ receptor function}

The idea that the Ins $\mathrm{P}_{3} \mathrm{R}$ can generate different classes of calcium signals, which result in specific cellular and systemic phenotypes, is not new. However, it has not been tested before in a systemic context. One possibility that we considered for the differential suppression of $i t p r$ phenotypes by $\mathrm{Kum}^{170}$ was that $\mathrm{InsP}_{3}$ is generated in the two contexts by the activation of either PLC $\gamma$ or $\mathrm{PLC} \beta$. Both these isoforms of PLC are known to generate $\mathrm{InsP}_{3}$, leading to activation of the $\mathrm{InsP}_{3} \mathrm{R}$ in vertebrates and inverte- brates (Rebecchi and Pentyala, 2000). Interestingly, our results in Drosophila do not support this idea, because none of the itpr mutant phenotypes was enhanced by reducing PLC $\gamma$ activity $(\mathrm{Ta}-$ ble 1). Rather, they suggest that activation of the $\operatorname{Ins}_{3} \mathrm{R}$ is primarily through Gq $\alpha$ and PLC $\beta$. All itpr mutant phenotypes, except for the maintenance of flight patterns, are enhanced by the $d g q$ alleles described here. Because an extended flight pattern is also the one phenotype that cannot be suppressed by $\mathrm{Kum}^{170}$, we attribute this phenotype to an intrinsic property of the $\operatorname{Ins}_{3} \mathrm{R}$.

From suppression of the $d g q$ - and plc21C-mediated enhancement of $i t p r$ phenotypes by $\mathrm{Kum}^{170}$, we can be reasonably certain that changes in cytosolic $\mathrm{Ca}^{2+}$ levels are the basis of all the phenotypes. Our data thus support the idea that $\operatorname{InsP}_{3}$-mediated $\mathrm{Ca}^{2+}$ release is further compromised in itpr mutants by the introduction of $d g q$ and $p l c 21 c$ alleles. However, we cannot rule out the possibility that $d g q$ and $p l c 21 c$ mutants act through a parallel pathway such as the generation of DAG and reduced $\mathrm{Ca}^{2+}$ entry through a DAG-dependent plasma membrane $\mathrm{Ca}^{2+}$ channel (Hardie, 2003). Additional interaction studies with mutants in this arm of the pathway are required to test this possibility rigorously.

\section{InsP $\mathrm{P}_{3}$-mediated $\mathrm{Ca}^{2+}$ release and development of the flight circuit}

Our analysis of flight in itpr mutants has demonstrated that InsP $\mathrm{P}_{3}$ signaling in the DdcGAL4 domain, which consists primarily of aminergic interneurons, is necessary for development of the flight circuit (Banerjee et al., 2004). From loss of the rhythmic response to an air puff in itpr mutants, it seems likely that neurons sensitive to Ins $\mathrm{P}_{3}$ signaling form part of the central pattern generator (CPG) for flight in Drosophila. Based on the genetic and phenotypic analysis presented here, we now propose that there are several steps in the formation of the flight CPG, which are differentially sensitive to $\mathrm{Ins}_{3}$ signaling. For example, the suppression of increased spontaneous firing, recorded from the DLMs of itpr mutants by the introduction of $\mathrm{Kum}^{170}$, is one such step. The importance of rhythmic spontaneous electrical activity in the development of activity in neural circuits is well known (Zhang and Poo, 2001). Another step is the connection(s) required for initiating flight patterns. Finally, to obtain normal air puff-induced flight, appropriate connectivity is necessary to maintain flight patterns for longer periods of $30 \mathrm{~s}$ or more. From the genetic paradigm used here, it is clear that the nature of the Ins $\mathrm{P}_{3}$ signal at this step is distinct from the others. One possibility is that it requires a specific frequency and/or amplitude of calcium waves, which are dependent on the intrinsic calcium release properties of the $\mathrm{InsP}_{3} \mathrm{R}$ (supplemental Fig. 1, available at www. jneurosci.org as supplemental material). Elucidating what each step and phenotype denotes in terms of CPG connectivity and how this is altered in itpr mutants, is a major challenge and is likely to require several different approaches in the future.

\section{References}

Ashburner M (1989) Drosophila, a laboratory handbook. Cold Spring Harbor, NY: Cold Spring Harbor Laboratory.

Banerjee S, Lee J, Venkatesh K, Wu CF, Hasan G (2004) Loss of flight and associated neuronal rhythmicity in inositol 1,4,5-trisphosphate receptor mutants of Drosophila. J Neurosci 24:7869-7878.

Benzer S (1973) Genetic dissection of behavior. Sci Am 229:24-37.

Berridge MJ (1993) Inositol trisphosphate and calcium signalling. Nature 361:315-325.

Berridge MJ (1998) Neuronal calcium signaling. Neuron 21:13-26.

Berridge MJ, Bootman MD, Roderick HL (2003) Calcium signalling: dynamics, homeostasis and remodelling. Nat Rev Mol Cell Biol 4:517-529. 
Bloomquist BT, Shortridge RD, Schneuwly S, Perdew M, Montell C, Steller H, Rubin G, Pak WL (1988) Isolation of a putative phospholipase C gene of Drosophila, norpA, and its role in phototransduction. Cell 54:723-733.

Borodinsky LN, Root CM, Cronin JA, Sann SB, Gu X, Spitzer NC (2004) Activity-dependent homeostatic specification of transmitter expression in embryonic neurons. Nature 429:523-530.

Bramley TA, Menzies GS, Jowett T (1990) Specific binding sites for ${ }^{125} \mathrm{I}-$ labelled human luteinizing hormone (hLH) in microsomal fractions from Drosophila. Mol Cell Endocrinol 73:93-104.

Brand AH, Manoukian AS, Perrimon N (1994) Ectopic expression in Drosophila. Methods Cell Biol 44:635-654.

De Koninck P, Schulman H (1998) Sensitivity of CaM kinase II to the frequency of $\mathrm{Ca}^{2+}$ oscillations. Science 279:227-230.

Dolmetsch RE, Lewis RS, Goodnow CC, Healy JI (1997) Differential activation of transcription factors induced by $\mathrm{Ca}^{2+}$ response amplitude and duration. Nature 386:855-858.

Dolmetsch RE, Xu K, Lewis RS (1998) Calcium oscillations increase the efficiency and specificity of gene expression. Nature 392:933-936.

Duch C, Levine RB (2002) Changes in calcium signaling during postembryonic dendritic growth in Manduca sexta. J Neurophysiol 87:1415-1425.

Engel JE, Wu CF (1992) Interactions of membrane excitability mutations affecting potassium and sodium currents in the flight and giant fiber escape systems of Drosophila. J Comp Physiol A Neuroethol Sens Neural Behav Physiol 171:93-104.

Ginzburg L, Futerman AH (2005) Defective calcium homeostasis in the cerebellum in a mouse model of Niemann-Pick A disease. J Neurochem 95:1619-1628.

Gomez TM, Zheng JQ (2006) The molecular basis for calcium-dependent axon pathfinding. Nat Rev Neurosci 7:115-125.

Hardie RC (2003) Regulation of TRP channels via lipid second messengers. Annu Rev Physiol 65:735-759.

Hille B, Tse A, Tse FW, Almers W (1994) Calcium oscillations and exocytosis in pituitary gonadotropes. Ann NY Acad Sci 710:261-270.

Joshi R, Venkatesh K, Srinivas R, Nair S, Hasan G (2004) Genetic dissection of itpr gene function reveals a vital requirement in aminergic cells of Drosophila larvae. Genetics 166:225-236.

Lee J, Wu CF (2002) Electroconvulsive seizure behavior in Drosophila: analysis of the physiological repertoire underlying a stereotyped action pattern in bang-sensitive mutants. J Neurosci 22:11065-11079.

Lee YJ, Dobbs MB, Verardi ML, Hyde DR (1990) dgq: a Drosophila gene encoding a visual system-specific $\mathrm{G} \alpha$ molecule. Neuron 5:889-898.

Lee YJ, Shah S, Suzuki E, Zars T, O’Day PM, Hyde DR (1994) The Drosophila dgq gene encodes a $\mathrm{G} \alpha$ protein that mediates phototransduction. Neuron 13:1143-1157.

Li H, Chaney S, Roberts IJ, Forte M, Hirsh J (2000) Ectopic G-protein expression in dopamine and serotonin neurons blocks cocaine sensitization in Drosophila melanogaster. Curr Biol 10:211-214.

Lin X, Antalffy B, Kang D, Orr HT, Zoghbi HY (2000) Polyglutamine expansion down-regulates specific neuronal genes before pathologic changes in SCA1. Nat Neurosci 3:157-163.

Ludwig M, Sabatier N, Bull PM, Landgraf R, Dayanithi G, Leng G (2002) Intracellular calcium stores regulate activity-dependent neuropeptide release from dendrites. Nature 418:85-89.

Manning CM, Mathews WR, Fico LP, Thackeray JR (2003) Phospholipase $\mathrm{C}-\gamma$ contains introns shared by src homology 2 domains in many unrelated proteins. Genetics 164:433-442.

Mason MJ, Garcia-Rodriguez C, Grinstein S (1991) Coupling between intracellular $\mathrm{Ca}^{2+}$ stores and the $\mathrm{Ca}^{2+}$ permeability of the plasma membrane. Comparison of the effects of thapsigargin, 2,5-di-(tert-butyl)-1,4hydroquinone, and cyclopiazonic acid in rat thymic lymphocytes. J Biol Chem 266:20856-20862.
Pearn MT, Randall LL, Shortridge RD, Burg MG, Pak WL (1996) Molecular biochemical, and electrophysiological characterization of Drosophila norpA mutants. J Biol Chem 271:4937-4945.

Ratnaparkhi A, Banerjee S, Hasan G (2002) Altered levels of Gq activity modulate axonal pathfinding in Drosophila. J Neurosci 22:4499-4508.

Rebecchi MJ, Pentyala SN (2000) Structure, function, and control of phosphoinositide-specific phospholipase C. Physiol Rev 80:1291-1335.

Sanyal S, Consoulas C, Kuromi H, Basole A, Mukai L, Kidokoro Y, Krishnan KS, Ramaswami M (2005a) Analysis of conditional paralytic mutants in Drosophila sarco-endoplasmic reticulum calcium ATPase reveals novel mechanisms for regulating membrane excitability. Genetics 169:737-750.

Sanyal S, Jennings T, Dowse H, Ramaswami M (2005b) Conditional mutations in SERCA, the sarco-endoplasmic reticulum $\mathrm{Ca}^{2+}$-ATPase, alter heart rate and rhythmicity in Drosophila. J Comp Physiol [B]:1-11.

Scott K, Zuker CS (1998) Assembly of the Drosophila phototransduction cascade into a signalling complex shapes elementary responses. Nature 395:805-808

Scott K, Becker A, Sun Y, Hardy R, Zuker C (1995) Gq $\alpha$ protein function in vivo: genetic dissection of its role in photoreceptor cell physiology. Neuron 15:919-927.

Shortridge RD, Yoon J, Lending CR, Bloomquist BT, Perdew MH, Pak WL (1991) A Drosophila phospholipase C gene that is expressed in the central nervous system. J Biol Chem 266:12474-12480.

Spitzer NC (2002) Activity-dependent neuronal differentiation prior to synapse formation: the functions of calcium transients. J Physiol (Paris) 96:73-80.

Spitzer NC, Lautermilch NJ, Smith RD, Gomez TM (2000) Coding of neuronal differentiation by calcium transients. Bioessays 22:811-817.

Srikanth S, Wang Z, Tu H, Nair S, Mathew MK, Hasan G, Bezprozvanny I (2004a) Functional properties of the Drosophila melanogaster inositol 1,4,5-trisphosphate receptor mutants. Biophys J 86:3634-3646.

Srikanth S, Wang Z, Hasan G, Bezprozvanny I (2004b) Functional properties of a pore mutant in the Drosophila melanogaster inositol 1,4,5trisphosphate receptor. FEBS Lett 575:95-98.

Tanouye MA, Wyman RJ (1980) Motor outputs of giant nerve fiber in Drosophila. J Neurophysiol 44:405-421.

Thackeray JR, Gaines PC, Ebert P, Carlson JR (1998) small wing encodes a phospholipase C- $(\gamma)$ that acts as a negative regulator of R7 development in Drosophila. Development 125:5033-5042.

Thastrup O, Cullen PJ, Drobak BK, Hanley MR, Dawson AP (1990) Thapsigargin, a tumor promoter, discharges intracellular $\mathrm{Ca}^{2+}$ stores by specific inhibition of the endoplasmic reticulum $\mathrm{Ca}^{2+}$-ATPase. Proc Natl Acad Sci USA 87:2466-2470.

Thorn P, Lawrie AM, Smith PM, Gallacher DV, Petersen OH (1993a) Local and global cytosolic $\mathrm{Ca}^{2+}$ oscillations in exocrine cells evoked by agonists and inositol trisphosphate. Cell 74:661-668.

Thorn P, Lawrie AM, Smith PM, Gallacher DV, Petersen OH (1993b) $\mathrm{Ca}^{2+}$ oscillations in pancreatic acinar cells: spatiotemporal relationships and functional implications. Cell Calcium 14:746-757.

Venkatesh K, Siddhartha G, Joshi R, Patel S, Hasan G (2001) Interactions between the inositol 1,4,5-trisphosphate and cyclic AMP signaling pathways regulate larval molting in Drosophila. Genetics 158:309-318.

Weinkove D, Neufeld TP, Twardzik T, Waterfield MD, Leevers SJ (1999) Regulation of imaginal disc cell size, cell number and organ size by Drosophila class I(A) phosphoinositide 3-kinase and its adaptor. Curr Biol 9:1019-1029.

Wu CF, Suzuki N, Poo MM (1983) Dissociated neurons from normal and mutant Drosophila larval central nervous system in cell culture. J Neurosci 3:1888-1899.

Zhang LI, Poo MM (2001) Electrical activity and development of neural circuits. Nat Neurosci 4 [Suppl]:1207-1214. 